Research Article

\title{
State-Space Measurement Update for GNSS/INS Integrated Navigation
}

\author{
XingLi Gan, ${ }^{1,2,3}$ Wei $\mathrm{Li}^{4}{ }^{4}$ Ling Yang $\mathbb{D}^{1},{ }^{1}$ and Heng Zhang ${ }^{2,3}$ \\ ${ }^{1}$ College of Surveying and Geo-informatics, Tongji University, Shanghai 200092, China \\ ${ }^{2}$ State Key Laboratory of Satellite Navigation System and Equipment Technology, Shijiazhuang 050081, China \\ ${ }^{3}$ The 54th Research Institute of China Electronics Technology Group Corporation, Shijiazhuang 050081, China \\ ${ }^{4}$ Shanghai Institute of Satellite Engineering, Shanghai 200240, China
}

Correspondence should be addressed to Ling Yang; ling_yangsy@163.com

Received 21 July 2020; Accepted 24 August 2020; Published 27 September 2020

Guest Editor: Gaoge Hu

Copyright (C) 2020 XingLi Gan et al. This is an open access article distributed under the Creative Commons Attribution License, which permits unrestricted use, distribution, and reproduction in any medium, provided the original work is properly cited.

\begin{abstract}
This paper theoretically derives the equivalence conditions for the loosely and tightly coupled GNSS/INS integration algorithms. Firstly, the equivalence is proved when using single epoch GNSS measurements, which means the GNSS processor provides standalone solution. Then, the equivalence proof is further extended for the filtering solutions, which are usually applied for differential GNSS and precise point positioning. Based on these, different state and measurement models for GNSS/INS integration navigation are summarized, and natural differences among these models are discussed. This indicates that once the same measurement and predict information are used, the integration would be equivalent no matter what kind of coupling schemes are used. A flight dataset with GNSS and tactical IMU data is used to evaluate the equivalence and discrepancies among four different measurement models, and the results confirm the theoretical derivations.
\end{abstract}

\section{Introduction}

Although differential global navigation satellite system (DGNSS) and precise point positioning (PPP) techniques nowadays provide viable precise positioning options, the major disadvantage of GNSS still remains: signal blockage due to obstructions in urban and built-up environments and extreme power attenuation of the signals when operated indoors [1]. Integrated navigation systems employing GNSS with other sensors, such as a self-contained inertial navigation system (INS), have the potential to provide high levels of accuracy and integrity $[2,3]$. The integrated systems can not only mitigate the weakness of GNSS but also bound the INS error that otherwise would grow with time when the INS operates alone [4]. The presence of multiple data sources theoretically provides functional redundancy as well as greater observability of the desired navigation states $[5,6]$.

To achieve precise navigation, DGNSS or PPP are involved in the loosely coupled (LC) or tightly coupled
(TC) GNSS/INS integration [4, 7-11]. In the LC approach, the GNSS solution is computed firstly using only the GNSS measurements and then combined with the IMU data in a secondary processor $[2,4]$. When less than 4 satellites are visible, a GNSS solution is not possible and the LC integration processor will be processed in a free-inertial model without GNSS position or velocity updates to constrain the inertial drift $[12,13]$. The TC approach directly combines the GNSS pseudorange, pseudorange-rate, and carrier phase measurements with the inertial measurements in an extended Kalman filter [14]. Therefore, the data utilization is better than the LC approach, and carrier phase ambiguities could be reinitialized for short GPS outages much more quickly. It has been agreed that the system reliability and faulttolerance are improved with the TC approach $[10,15$, 16]. In recent decades, tightly coupled DGNSS/INS or $\mathrm{PPP} / \mathrm{INS}$ integration has become popular, because it has the advantage of providing accurate position information even when GNSS measurements are not enough for 
standalone processing and is theoretically optimal in a filtering sense, especially in urban navigation applications $[8,16]$. PPP using ionosphere-free combination or ionosphere-constraint combination has been introduced into tightly coupled integration with INS $[15,17]$. Constraints such as context features, GNSS baseline length, vehicle moving properties have also been considered to improve the observability, reliability, stability, and availability of the GNSS/INS integration systems $[1,2,4,9,18]$. However, the heavier computational burden and more data exchange among subsystems usually complicate the TC processing and reduce the efficiency.

In this paper, we proposed a unified integration mode, with the measurement update based on the state space, by which the LC and TC processors are united and both the system efficiency and precision can be guaranteed. The paper is organised as follows. The general dynamic and measurement model of GNSS/INS integration algorithms is described in Section 2. Then, in Section 3, we theoretically construct the state-based measurement update model based on the equivalence derivation between the LC and TC approach involving single epoch GNSS measurements. The state-based measurement update approach is further extended when time correlations are considered in the GNSS processor in Section 4. Based on this, the possible equivalent models for GNSS/INS integration are summarized. Experiment results are presented to demonstrate the theoretical derivation in Section 5. Finally, some concluding remarks are presented in Section 6.

\section{Full State for GNSS/INS Integration Algorithms}

2.1. Full State Model Including All Parameters. For GNSS/ INS integration processing, the following states can be consisted in the integration state vector as given in Table 1.

For the GNSS positioning model, the general full state vector is

$$
\mathbf{x}_{G}=\left[\begin{array}{lll}
\mathbf{x}_{\mathrm{vp}, G}^{T} & \mathbf{x}_{b}^{T} & \mathbf{x}_{c}^{T}
\end{array}\right]^{T},
$$

and for the GNSS/INS integration model, the general full state vector is

$$
\mathbf{x}=\left[\begin{array}{lllll}
\mathbf{x}_{\mathrm{avp}, I}^{T} & \mathbf{x}_{\mathrm{imu}}^{T} & \mathbf{x}_{\eta}^{T} & \mathbf{x}_{b}^{T} & \mathbf{x}_{c}^{T}
\end{array}\right]^{T},
$$

where variables in (1) and (2) are explained in Table 1.

2.2. Dynamic and Measurement Model for Kalman Filter. The system error dynamics of GNSS/INS integration are based on the INS error equations [19]. The psi-angle error equations of INS are expressed in the navigation frame ( $n$ frame, local east, north, and up) for position, velocity, and attitude updating [20-22].

$$
\left\{\begin{array}{l}
\dot{x}_{p}^{n}=-\boldsymbol{\omega}_{\mathrm{en}}^{n} \times \mathbf{x}_{p}^{n}+\mathbf{x}_{v}^{n}, \\
\dot{x}_{v}^{n}=-\left(\boldsymbol{\omega}_{\mathrm{ie}}^{n}+\boldsymbol{\omega}_{\mathrm{in}}^{n}\right) \times \mathbf{x}_{v}^{n}-\boldsymbol{\psi} \times \mathbf{f}^{n}+\delta \mathbf{g}^{n}+\mathbf{C}_{b}^{n} \mathbf{x}_{\nabla}, \\
\dot{\psi}=\boldsymbol{\psi} \times \boldsymbol{\omega}_{\text {in }}^{n}-\mathbf{C}_{b}^{n} \mathbf{x}_{\varepsilon},
\end{array}\right.
$$

where the superscript $(n)$ refers to the navigation frame ( $n$ frame), while the subscripts $(i)$ and $(e)$ denote the inertial and the earth-centred earth-fixed frame ( $i$-frame and $e$ frame), respectively; $\mathbf{x}_{p}^{n}, \mathbf{x}_{v}^{n}$, and $\psi$ are, respectively, the position, velocity, and attitude error vector; $\omega_{\mathrm{ie}}^{n}$ is the earth rotation angular rate; $\omega_{\mathrm{in}}^{n}$ is the angular rate of the navigation coordinate system with respect to the $i$-frame; $\mathbf{f}^{n}$ is the specific force vector; $\delta \mathbf{g}^{n}$ is the error of the gravity vector in the $n$-frame; $\omega_{\text {en }}^{n}$ is the angular rate from the $e$-frame to the $n$ frame; $\mathbf{C}_{b}^{n}$ is the coordinate transformation matrix from body frame ( $b$-frame) to $n$-frame; and $\mathbf{x}_{\nabla}$ and $\mathbf{x}_{\varepsilon}$ are the accelerometer and gyro drift vector expressed in the $b$-frame, respectively.

The Kalman filter (KF) algorithm uses the measurement vector, measurement model, and dynamic model to maintain optimal estimates of the state vector. The general KF dynamic model is given as

$$
\dot{x}_{k}=\mathbf{F}_{k} \mathbf{x}_{k}+\mathbf{w}_{k},
$$

where $\mathbf{w}_{k}$ is the system noise vector comprising a number of independent random noise sources and $\mathbf{F}_{k}$ is the transition matrix derived from the known properties of the system.

With the dynamic model describing the properties of the system's dynamic, the KF measurement model describes the functional relationship between the measurement vector and the state vector and is generally given as

$$
\begin{aligned}
\mathbf{z}_{k} & =\mathbf{H}_{k} \mathbf{x}_{k}+\boldsymbol{\varepsilon}_{k}, \\
\boldsymbol{\varepsilon}_{k} & \sim \mathbf{N}\left(0, \mathbf{R}_{k}\right),
\end{aligned}
$$

where subscript $k$ is the epoch indicator; $\mathbf{z}_{k} \in \mathbb{R}^{m}$ is the measurement vector; $\mathrm{H}_{k} \in \mathbb{R}^{m \times f}$ is the design matrix relating the measurements to the state parameters; and $\varepsilon_{k}$ is the measurement noise vector with a covariance matrix $\mathbf{R}_{k}$.

The KF measurement update is triggered at every GNSS measurement epoch using the difference between the GNSS and the INS mechanization solution. In LC mode, the states estimated from the GNSS processor are used as measurements in KF. In TC mode, the GNSS pseudorange, pseudorange-rate, and carrier phase measurements are directly used as measurements in KF.

The measurements for LC integration are the position and velocity difference between GPS and INS centre and are given as

$$
\mathbf{z}_{k}=\left[\begin{array}{c}
\mathbf{X}_{p}^{*}-\mathbf{X}_{p, G}^{*} \\
\mathbf{X}_{v}^{*}-\mathbf{X}_{v, G}^{*}
\end{array}\right]
$$

where superscript $*$ means the state-resolved frame and variants are explained in Table 1.

The solution of the above system, (4) and (5), can be expressed by prediction and measurement update (e.g., see [5]). The prediction of the state and its covariance matrix are 
TABLE 1: Full error state vector in GNSS/INS integration.

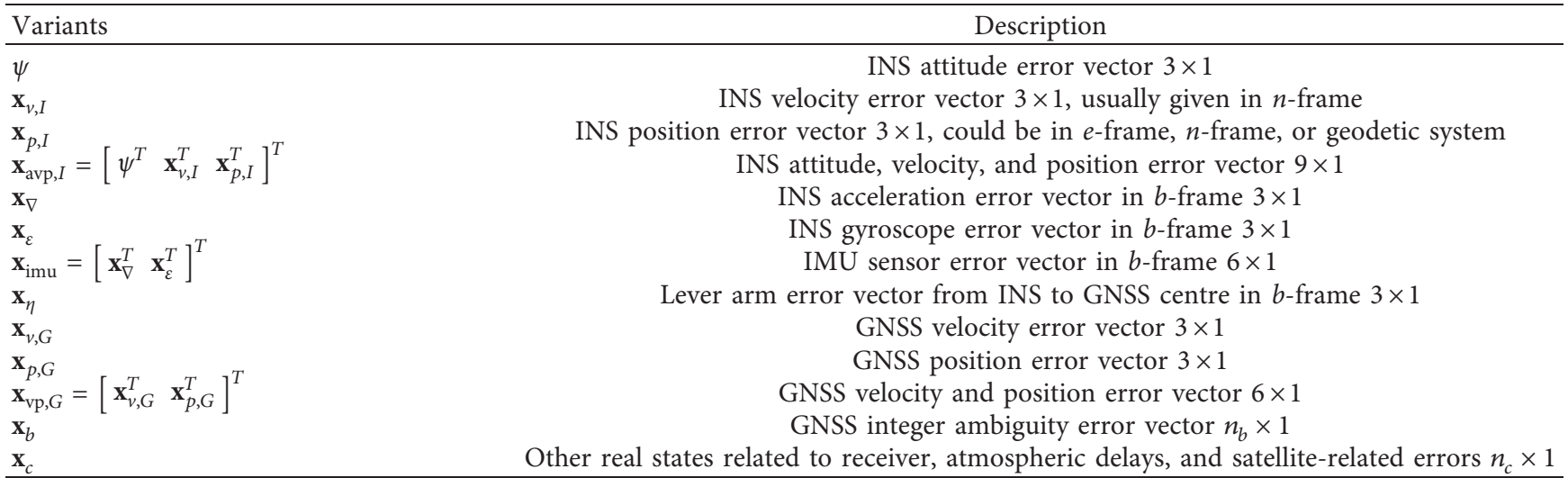

$$
\left\{\begin{array}{l}
\overline{\mathbf{x}}_{k}=\boldsymbol{\Phi}_{k} \widehat{\mathbf{x}}_{k-1}, \\
\overline{\mathbf{P}}_{k}=\boldsymbol{\Phi}_{k} \mathbf{P}_{k-1} \boldsymbol{\Phi}_{k}^{T}+\mathbf{Q}_{k},
\end{array}\right.
$$

where $\overline{\mathbf{x}}_{k}$ is the state prediction; $\widehat{\mathbf{x}}_{k-1}$ is the state estimate at the previous epoch; $\Phi_{k} \approx \mathbf{I}+\mathbf{F} \Delta t+\left(\mathbf{F}(\Delta t)^{2} / 2 !\right)+$ is the state transition matrix; $\mathbf{P}_{k-1}$ is the covariance matrix of $\widehat{\mathbf{x}}_{k-1} ; \mathbf{Q}_{k}$ is the dynamic noise covariance matrix; and $\overline{\mathbf{P}}_{k}$ is the covariance matrix of the prediction $\overline{\mathbf{x}}_{k}$. When the measurements are available, the state vector is updated. The Kalman gain $\mathbf{K}_{k}$, the updated state, and its covariance matrix are given by

$$
\left\{\begin{array}{l}
\mathbf{K}_{k}=\overline{\mathbf{P}}_{k} \mathbf{H}_{k}^{T}\left(\mathbf{H}_{k} \overline{\mathbf{P}}_{k} \mathbf{H}_{k}^{T}+\mathbf{R}_{k}\right)^{-1}=\mathbf{P}_{k} \mathbf{H}_{k}^{T} \mathbf{R}_{k}^{-1}, \\
\widehat{\mathbf{x}}_{k}=\overline{\mathbf{x}}_{k}+\mathbf{K}_{k}\left(\mathbf{z}_{k}-\mathbf{H}_{k} \overline{\mathbf{x}}_{k}\right), \\
\mathbf{P}_{k}=\left(\mathbf{I}-\mathbf{K}_{k} \mathbf{H}_{k}\right) \overline{\mathbf{P}}_{k}=\left(\mathbf{H}_{k} \mathbf{R}_{k}^{-1} \mathbf{H}_{k}^{T}+\overline{\mathbf{P}}_{k}^{-1}\right)^{-1},
\end{array}\right.
$$

where $\widehat{\mathbf{x}}_{k}$ is the KF estimate of the state and $\mathbf{P}_{k}$ is the corresponding estimation covariance matrix.

\section{State-Based Measurement Model for GNSS/ INS Integration}

With the utilization of multiconstellation GNSS, integration measurements and states would increase dramatically and accordingly increase the date exchange and computation burden. To simplify the integration structure, we only consider the INS-related states in the integration model as

$$
\mathbf{x}_{I}=\left[\begin{array}{lll}
\mathbf{x}_{\mathrm{avp}, I}^{T} & \mathbf{x}_{\mathrm{imu}}^{T} & \mathbf{x}_{\eta}^{T}
\end{array}\right]^{T} .
$$

(1)-(9) show that the common states between GNSS and INS include the position and velocity states, The relationship between $\mathbf{x}_{\mathrm{vp}, G}$ and $\mathbf{x}_{\mathrm{avp}}$ are $[23,24]$

$$
\left\{\begin{array}{l}
\mathbf{x}_{v, G}^{*}=\mathbf{x}_{v}^{*}+\mathbf{C}_{b}^{*}\left(\boldsymbol{\omega}_{\mathrm{eb}}^{b} \times\right) \mathbf{x}_{\eta}, \\
\mathbf{x}_{p, G}^{*}=\mathbf{x}_{p}^{*}+\mathbf{C}_{b}^{*} \mathbf{x}_{\eta},
\end{array}\right.
$$

where $\mathbf{C}_{b}^{*}$ is the transition matrix from $b$-frame to $*$-frame; $\omega_{\mathrm{eb}}^{b}$ is the angular rate of the $b$-frame respect to the $e$-frame, resolved in the $b$-frame; and superscript $*$ means the state- resolved frame and other variants are expressed in Table 1. The matrix format is

$$
\mathbf{x}_{\mathrm{vp}, G}=\mathbf{T}_{g, i} \mathbf{x}_{I}
$$

with

$$
\mathbf{T}_{g, i}=\left[\begin{array}{llll}
0_{6 \times 3} & \mathbf{I}_{6 \times 6} & 0_{6 \times 6} & \mathbf{T}_{v p, \eta}
\end{array}\right] .
$$

Usually, the velocity state $\mathbf{x}_{v}$ is solved in $n$-frame. If $\mathbf{x}_{p}$ and $\mathbf{x}_{p, G}$ are also given in $n$-frame, there is

$$
\mathbf{T}_{v p, \eta}=\left[\begin{array}{c}
\mathbf{C}_{b}^{n}\left(\boldsymbol{\omega}_{\mathrm{eb}}^{b} \times\right) \\
\mathbf{C}_{b}^{n}
\end{array}\right] .
$$

3.1. Measurement Model at Single Epoch. As the states given in (1), the measurement model of GNSS positioning can be written as

$$
\begin{aligned}
& \mathbf{l}=\mathbf{A} \mathbf{x}_{\mathrm{vp}, G}+\mathbf{B} \mathbf{x}_{b}+\mathbf{C} \mathbf{x}_{c}, \\
& \boldsymbol{\varepsilon} \sim \mathbf{N}(0, \boldsymbol{\Sigma}),
\end{aligned}
$$

where $\mathbf{l} \in \mathbb{R}^{m}$ is the measurement vector; $\mathbf{A} \in \mathbb{R}^{m \times 6}$, $\mathbf{B} \in \mathbb{R}^{m \times n_{b}}$, and $\mathbf{C} \in \mathbb{R}^{m \times n_{c}}$ are the design matrices related to the respective states; and $\mathbf{x}_{\mathrm{vp}, G}, \mathbf{x}_{b}$, and $\mathbf{x}_{c}$ are defined in Table 1. Without any prediction model and letting $\mathbf{N}_{I J}=\mathbf{I}^{T} \Sigma^{-1} \mathbf{J}, \mathbf{U}_{I}=\mathbf{I}^{T} \Sigma^{-1} \mathbf{l}$, the least-square norm equation of (14) is

$$
\left[\begin{array}{lll}
\mathbf{N}_{A A} & \mathbf{N}_{A B} & \mathbf{N}_{A C} \\
\mathbf{N}_{B A} & \mathbf{N}_{B B} & \mathbf{N}_{B C} \\
\mathbf{N}_{C A} & \mathbf{N}_{C B} & \mathbf{N}_{C C}
\end{array}\right]\left[\begin{array}{c}
\mathbf{x}_{\mathrm{vp}, G} \\
\mathbf{x}_{b} \\
\mathbf{x}_{c}
\end{array}\right]=\left[\begin{array}{c}
\mathbf{U}_{A} \\
\mathbf{U}_{B} \\
\mathbf{U}_{C}
\end{array}\right] .
$$

Using the reduction algorithm, norm equation (15) is equal to

$$
\left(\mathbf{M}_{A C}-\mathbf{M}_{A B C} \mathbf{M}_{B C}^{-1} \mathbf{M}_{B A C}\right) \mathbf{x}_{\mathrm{vp}, G}=\mathbf{U}_{A C}-\mathbf{M}_{A B C} \mathbf{M}_{B C}^{-1} \mathbf{U}_{B C},
$$


with

$$
\left\{\begin{array}{l}
\mathbf{M}_{A C}=\mathbf{N}_{A A}-\mathbf{N}_{A C} \mathbf{N}_{C C}^{-1} \mathbf{N}_{C A}, \\
\mathbf{M}_{A B C}=\mathbf{N}_{A B}-\mathbf{N}_{A C} \mathbf{N}_{C C}^{-1} \mathbf{N}_{C B}, \\
\mathbf{M}_{B C}=\mathbf{N}_{B B}-\mathbf{N}_{B C} \mathbf{N}_{C C}^{-1} \mathbf{N}_{C B}, \\
\mathbf{M}_{B A C}=\mathbf{N}_{B A}-\mathbf{N}_{B C} \mathbf{N}_{C C}^{-1} \mathbf{N}_{C A}, \\
\mathbf{U}_{A C}=\mathbf{U}_{A}-\mathbf{N}_{A C} \mathbf{N}_{C C}^{-1} \mathbf{U}_{C}, \\
\mathbf{U}_{B C}=\mathbf{U}_{B}-\mathbf{N}_{B C} \mathbf{N}_{C C}^{-1} \mathbf{U}_{C}
\end{array}\right.
$$

Combining (11) and (16), there is

$$
\begin{gathered}
\mathbf{T}_{g, i}^{T}\left(\mathbf{M}_{A C}-\mathbf{M}_{A B C} \mathbf{M}_{B C}^{-1} \mathbf{M}_{B A C}\right) \mathbf{T}_{g, i} \mathbf{x}_{I} \\
\quad=\mathbf{T}_{g, i}^{T}\left(\mathbf{U}_{A C}-\mathbf{M}_{A B C} \mathbf{M}_{B C}^{-1} \mathbf{U}_{B C}\right) .
\end{gathered}
$$

With predict model (7), the integration solution is

$$
\left\{\begin{array}{l}
\mathbf{P}=\mathbf{D}\left(\mathbf{x}_{I}\right)=\left(\mathbf{T}_{g, i}^{T}\left(\mathbf{M}_{A C}-\mathbf{M}_{A B C} \mathbf{M}_{B C}^{-1} \mathbf{M}_{B A C}\right) \mathbf{T}_{g, i}+\overline{\mathbf{P}}^{-1}\right)^{-1}, \\
\widehat{\mathbf{x}}_{I}=\mathbf{P}\left(\overline{\mathbf{P}}^{-1} \overline{\mathbf{x}}+\mathbf{T}_{g, i}^{T}\left(\mathbf{U}_{A C}-\mathbf{M}_{A B C} \mathbf{M}_{B C}^{-1} \mathbf{U}_{B C}\right)\right) .
\end{array}\right.
$$

To unify the state with INS, the measurement model containing full state is

$$
\begin{aligned}
& \mathbf{1}=\mathbf{A T}_{g, i} \mathbf{x}_{I}+\mathbf{B} \mathbf{x}_{b}+\mathbf{C} \mathbf{x}_{c}, \\
& \boldsymbol{\varepsilon} \sim \mathbf{N}(0, \boldsymbol{\Sigma}) .
\end{aligned}
$$

Without any prediction model and letting $\overline{\mathbf{A}}=\mathbf{A T}_{g, i}$ $\mathbf{N}_{I J}=\mathbf{I}^{T} \Sigma^{-1} \mathbf{J}, \mathbf{U}_{I}=\mathbf{I}^{T} \Sigma^{-1} \mathbf{l}$, the least-square norm equation of $(20)$ is

$$
\left[\begin{array}{lll}
\mathbf{N}_{\overline{A A}} & \mathbf{N}_{\bar{A} B} & \mathbf{N}_{\bar{A} C} \\
\mathbf{N}_{B \bar{A}} & \mathbf{N}_{B B} & \mathbf{N}_{B C} \\
\mathbf{N}_{C \bar{A}} & \mathbf{N}_{C B} & \mathbf{N}_{C C}
\end{array}\right]\left[\begin{array}{l}
\mathbf{x}_{I} \\
\mathbf{x}_{b} \\
\mathbf{x}_{c}
\end{array}\right]=\left[\begin{array}{l}
\mathbf{U}_{\bar{A}} \\
\mathbf{U}_{B} \\
\mathbf{U}_{C}
\end{array}\right]
$$

Without enough measurements, the coefficient matrix of the norm function could be ill-conditioned and the state vector is unsolvable or can only be solved with poor precision.

In (9), only position and velocity states are considered in the integration model; the dynamic model of state $\mathbf{x}_{b}$ and $\mathbf{x}_{c}$ should be considered within the GNSS processor. Supposing the corresponding prediction is $\overline{\mathbf{x}}_{b}, \overline{\mathbf{P}}_{b}$ and $\overline{\mathbf{x}}_{c}, \overline{\mathbf{P}}_{c}$, with $\overline{\mathbf{P}}_{*}$ denoting the corresponding predict variance matrix, and letting $\mathbf{N}_{I J}=\mathbf{N}_{I J}+\overline{\mathbf{P}} \overline{\mathbf{U}}_{I}=\mathbf{U}_{I}+\overline{\mathbf{P}}^{-1} \overline{\mathbf{x}},(21)$ is extended as

$$
\left[\begin{array}{ccc}
\overline{\mathbf{N}}_{\overline{A A}} & \mathbf{N}_{\bar{A} B} & \mathbf{N}_{\bar{A} C} \\
\mathbf{N}_{B \bar{A}} & \overline{\mathbf{N}}_{B B} & \mathbf{N}_{B C} \\
\mathbf{N}_{C \bar{A}} & \mathbf{N}_{C B} & \overline{\mathbf{N}}_{C C}
\end{array}\right]\left[\begin{array}{c}
\mathbf{x}_{\mathrm{vp}, G} \\
\mathbf{x}_{b} \\
\mathbf{x}_{c}
\end{array}\right]=\left[\begin{array}{c}
\overline{\mathbf{U}}_{\bar{A}} \\
\overline{\mathbf{U}}_{B} \\
\overline{\mathbf{U}}_{C}
\end{array}\right] .
$$

Using the reduction algorithm, norm equation (22) is equal to

$$
\left(\mathbf{M}_{\bar{A} C}-\mathbf{M}_{A B C} \mathbf{M}_{B C}^{-1} \mathbf{M}_{B A C}\right) \mathbf{x}_{I}=\mathbf{U}_{A C}-\mathbf{M}_{A B C} \mathbf{M}_{B C}^{-1} \mathbf{U}_{B C},
$$

with

$$
\left\{\begin{array}{l}
\mathbf{M}_{A C}=\overline{\mathbf{N}}_{A A}-\mathbf{N}_{A C} \overline{\mathbf{N}}_{C C}^{-1} \mathbf{N}_{C A}, \\
\mathbf{M}_{A B C}=\mathbf{N}_{A B}-\mathbf{N}_{A C} \overline{\mathbf{N}}_{C C}^{-1} \mathbf{N}_{C B}, \\
\mathbf{M}_{B C}=\overline{\mathbf{N}}_{B B}-\mathbf{N}_{B C} \overline{\mathbf{N}}_{C C}^{-1} \mathbf{N}_{C B}, \\
\mathbf{M}_{B A C}=\mathbf{N}_{B A}-\mathbf{N}_{B C} \overline{\mathbf{N}}_{C C}^{-1} \mathbf{N}_{C A}, \\
\mathbf{U}_{A C}=\mathbf{U}_{A}-\mathbf{N}_{A C} \overline{\mathbf{N}}_{C C}^{-1} \mathbf{U}_{C}, \\
\mathbf{U}_{B C}=\mathbf{U}_{B}-\mathbf{N}_{B C} \overline{\mathbf{N}}_{C C}^{-1} \mathbf{U}_{C} .
\end{array}\right.
$$

To investigate the solution difference between loosely and tightly coupled integration, the general mathematical model for GNSS positioning is given as

$$
\mathbf{l}=\mathbf{A x}_{G}+\boldsymbol{\varepsilon}, \quad \varepsilon \sim \mathbf{N}(0, \boldsymbol{\Sigma}),
$$

where $\mathbf{x}_{G}$ is the unknown state vector; $\mathbf{l}$ is the measurement vector corrected by an initial state va; and $\varepsilon$ is the measurement noise vector which is usually modelled as random noise. The least-squares solution is

$$
\left\{\begin{array}{l}
\widehat{\mathbf{x}}_{G}=\mathbf{N}^{-1} \mathbf{A}^{T} \boldsymbol{\Sigma}^{-1} \mathbf{l}, \\
\mathbf{N}=\mathbf{A}^{T} \boldsymbol{\Sigma}^{-1} \mathbf{A} \\
\mathbf{D}\left(\widehat{\mathbf{x}}_{G}\right)=\mathbf{N}^{-1}
\end{array}\right.
$$

Least-squares solution (26) will be used as the measurement model of LC integration, and mathematical model (25) will be used for the measurement model of TC integration. In the following subsection, the equivalence or discrepancy between loosely and tightly coupled algorithms will be derived.

3.2. Equivalence Proof between Loosely and Tightly Coupled Integration for GNSS Single Epoch Solution. With subscript "LC" denoting the LC scheme, the corresponding measurement model is

$$
\mathbf{z}_{k, \mathrm{LC}}=\mathbf{H}_{k, \mathrm{LC}} \mathbf{x}+\boldsymbol{\varepsilon}_{k, L C}
$$

It is derived from the difference of the GNSS solution and INS solution. From (10), there is

$$
\left\{\begin{array}{l}
\mathbf{X}_{v, G}^{n}=\mathbf{X}_{v, 0}^{n}+\mathbf{C}_{b}^{e}\left(\boldsymbol{\omega}_{e b}^{b} \times\right) \mathbf{X}_{\eta, 0}+\mathbf{x}_{v}^{n}+\mathbf{C}_{b}^{e}\left(\boldsymbol{\omega}_{e b}^{b} \times\right) \mathbf{x}_{\eta}, \\
\mathbf{X}_{p, G}^{e}=\mathbf{X}_{p, 0}^{e}+C_{b}^{e} \mathbf{X}_{\eta, 0}+\mathbf{x}_{p}^{e}+\mathbf{C}_{b}^{e} \mathbf{x}_{\eta}, \\
\mathbf{X}_{b}=\mathbf{X}_{b, 0}+\mathbf{x}_{b} .
\end{array}\right.
$$

The corresponding vector form is

$$
\left[\begin{array}{c}
\mathbf{X}_{v, G}^{n}-\mathbf{X}_{v, 0}^{n}-\mathbf{C}_{b}^{e}\left(\boldsymbol{\omega}_{\mathrm{eb}}^{b} \times\right) \mathbf{X}_{\eta, 0} \\
\mathbf{X}_{p, G}^{e}-\mathbf{X}_{p, 0}^{e}-C_{b}^{e} \mathbf{X}_{\eta, 0} \\
\mathbf{X}_{b}-\mathbf{X}_{b, 0}
\end{array}\right]=\left[\begin{array}{c}
\mathbf{x}_{v}^{n}+\mathbf{C}_{b}^{e}\left(\boldsymbol{\omega}_{\mathrm{eb}}^{b} \times\right) \mathbf{x}_{\eta} \\
\mathbf{x}_{p}^{e}+\mathbf{C}_{b}^{e} \mathbf{x}_{\eta} \\
\mathbf{x}_{b}
\end{array}\right]
$$

Therefore, it is derived as 


$$
\begin{aligned}
\mathbf{z}_{k, \mathrm{LC}} & =\left[\begin{array}{c}
\mathbf{X}_{v, G}^{n} \\
\mathbf{X}_{p, G}^{e} \\
\mathbf{X}_{b}
\end{array}\right]-\left[\begin{array}{c}
\mathbf{X}_{v, 0}^{n} \\
\mathbf{X}_{p, 0}^{e} \\
\mathbf{X}_{b, 0}
\end{array}\right]-\left[\begin{array}{c}
\mathbf{T}_{v p, \eta} \\
0_{n_{b} \times 3}
\end{array}\right] \mathbf{X}_{\eta, 0}, \\
\mathbf{H}_{k, \mathrm{LC}} & =\mathbf{T}_{g, i} .
\end{aligned}
$$

GNSS positioning processing requires for an approximate value of the state. In a GNSS/INS integration procedure, the approximate value can come from the INS time update and is given as

$$
\left[\begin{array}{c}
\mathbf{X}_{v, G, 0}^{n} \\
\mathbf{X}_{p, G, 0}^{e} \\
\mathbf{X}_{b, 0}
\end{array}\right]=\left[\begin{array}{c}
\mathbf{X}_{v, 0}^{n} \\
\mathbf{X}_{p, 0}^{e} \\
\mathbf{X}_{b, 0}
\end{array}\right]+\left[\begin{array}{c}
\mathbf{T}_{v p, \eta} \\
0
\end{array}\right] \mathbf{X}_{\eta, 0}
$$

Therefore,

$$
\left[\begin{array}{c}
\mathbf{X}_{v, G}^{n} \\
\mathbf{X}_{p, G}^{e} \\
\mathbf{X}_{b}
\end{array}\right]=\left[\begin{array}{c}
\mathbf{X}_{v, G, 0}^{n} \\
\mathbf{X}_{p, G, 0}^{e} \\
\mathbf{X}_{b, 0}^{e}
\end{array}\right]+\widehat{\mathbf{x}}_{G}=\left[\begin{array}{c}
\mathbf{X}_{v, 0}^{n} \\
\mathbf{X}_{p, 0}^{e} \\
\mathbf{X}_{b, 0}
\end{array}\right]+\left[\begin{array}{c}
\mathbf{T}_{\mathrm{vp}, \eta} \\
0
\end{array}\right] \mathbf{X}_{\eta, 0}+\widehat{\mathbf{x}}_{G},
$$

where $\widehat{\mathbf{x}}_{G}$ is from (26). Substituting (33) into (30), there is

$$
\begin{aligned}
& \mathbf{z}_{k, \mathrm{LC}}=\widehat{\mathbf{x}}_{G}, \\
& \mathbf{R}_{k, \mathrm{LC}}=\mathbf{D}\left(\widehat{\mathbf{x}}_{G}\right)=\mathbf{N}^{-1}=\left(\begin{array}{lll}
\mathbf{A}^{T} & \mathbf{\Sigma}^{-1} & \mathbf{A}
\end{array}\right)^{-1} .
\end{aligned}
$$

From (8), the LC integration solution is

$$
\left\{\begin{array}{l}
\mathbf{P}_{k, \mathrm{LC}}=\left(\mathbf{H}_{k, \mathrm{LC}}^{T} \mathbf{R}_{k, \mathrm{LC}}^{-1} \mathbf{H}_{k, \mathrm{LC}}+\overline{\mathbf{P}}_{k}^{-1}\right)^{-1}=\left(\mathbf{T}_{g, i}^{T} \mathbf{N} \mathbf{T}_{g, i}+\overline{\mathbf{P}}_{k}^{-1}\right)^{-1}, \\
\mathbf{K}_{k, \mathrm{LC}}=\mathbf{P}_{k, \mathrm{LC}} \mathbf{H}_{k, \mathrm{LC}}^{T} \mathbf{R}_{k, \mathrm{LC}}^{-1}=\mathbf{P}_{k, \mathrm{LC}} \mathbf{T}_{g, i}^{T} \mathbf{N}, \\
\widehat{\mathbf{x}}_{k, \mathrm{LC}}=\mathbf{K}_{k, \mathrm{LC}} \mathbf{z}_{k, \mathrm{LC}}=\mathbf{P}_{k, \mathrm{LC}} \mathbf{T}_{g, i}^{T} \mathbf{N} \widehat{x}_{G}=\mathbf{P}_{k, \mathrm{LC}} \mathbf{T}_{g, i}^{T} \mathbf{A}^{T} \boldsymbol{\Sigma}^{-1} \mathbf{l} .
\end{array}\right.
$$

From (25) and (26), the corresponding TC measurement model is

$$
\mathbf{l}=\mathbf{A T}_{g, i} \mathbf{x}+\boldsymbol{\varepsilon}, \quad \boldsymbol{\varepsilon} \sim \mathbf{N}(0, \boldsymbol{\Sigma}) .
$$

Therefore, the design matrix is

$$
\mathbf{H}_{k, \mathrm{TC}}=\mathbf{A T}_{g, i},
$$

and the corrected measurement vector is

$$
\mathbf{z}_{k, \mathrm{TC}}=\mathbf{1},
$$

with its variance being

$$
\mathbf{R}_{k, \mathrm{TC}}=\boldsymbol{\Sigma} .
$$

According to (38), (39), and (40), the TC integration solution is

$$
\left\{\begin{aligned}
\mathbf{P}_{k, \mathrm{TC}} & =\left(\mathbf{H}_{k, 2 \mathrm{TC}}^{T} \mathbf{R}_{k, \mathrm{TC}}^{-1} \mathbf{H}_{k, \mathrm{TC}}+\overline{\mathbf{P}}_{k}^{-1}\right)^{-1} \\
& =\left(\mathbf{T}_{g, i}^{T} \mathbf{N T}_{g, i}+\overline{\mathbf{P}}_{k}^{-1}\right)^{-1}=\mathbf{P}_{k, \mathrm{LC}}, \\
\mathbf{K}_{k, \mathrm{TC}} & =\mathbf{P}_{k, \mathrm{TC}} \mathbf{H}_{k, \mathrm{TC}}^{T} \mathbf{R}_{k, \mathrm{TC}}^{-1}=\mathbf{P}_{k, \mathrm{LC}} \mathbf{T}_{g, i}^{T} \mathbf{A}^{T} \boldsymbol{\Sigma}^{-1}, \\
\widehat{\mathbf{x}}_{k, \mathrm{TC}} & =\mathbf{K}_{k, \mathrm{TC}} \mathbf{z}_{k, \mathrm{TC}}=\mathbf{P}_{k, \mathrm{TC}} \mathbf{T}_{g, i}^{T} \mathbf{A}^{T} \boldsymbol{\Sigma}^{-1} \mathbf{l}=\widehat{\mathbf{x}}_{k, \mathrm{LC}},
\end{aligned}\right.
$$

(36) and (41) reveal that the loosely and tightly coupled schemes are equivalent in terms of the solution of $\widehat{\mathbf{x}}_{k}$ and the estimated variance $\mathbf{P}_{k}$, while the gain matrix $\mathbf{K}_{k}$ satisfied that

$$
\mathbf{K}_{k, \mathrm{LC}}=\mathbf{K}_{k, \mathrm{TC}} \mathbf{A} \text {. }
$$

It is noted that the above equivalence is satisfied based on three implied conditions: (1) in TC processor, the INS time update is used as the initial value for the linearization of the GNSS observation function; (2) the GNSS processor provides a standalone solution, which means no dynamic model used; (3) the measurement variances of LC and TC schemes are given by (35) and (40), respectively, which build the inner connection between them.

3.3. Equivalence Proof Based on Simplified Equivalent Equations at Single Epoch. Abovementioned general state function includes all parameters, while some of the parameters are not directly necessary for navigation, such as atmospheric parameters, GNSS receiver clock parameters, and ambiguities. Considering all parameters in the state vector would unavoidably increase the computation burden. Therefore, it is reasonable to utilize a simplified state model which only contains the public state in the integration filter. Dividing the unknown states of GNSS positioning into two parts, the navigation-related and navigation-unrelated (25) is rewritten as

$$
\mathbf{l}=\mathbf{A}_{1} \mathbf{x}_{\mathrm{vp}, G}+\mathbf{A}_{2} \mathbf{x}_{b}+\boldsymbol{\varepsilon}, \quad \boldsymbol{\varepsilon} \sim N(0, \boldsymbol{\Sigma}),
$$

where $\mathbf{x}_{\mathrm{vp}, G}$ denotes the GNSS position and velocity states as given in Table 1. By using the equivalent function, the above function can be rewritten as

$$
\mathbf{l}=\mathbf{R}_{1} \mathbf{A}_{1} \mathbf{x}_{\mathrm{vp}, G}+\varepsilon, \quad \varepsilon \sim \mathbf{N}(0, \Sigma),
$$

where $\quad \mathbf{R}_{1}=\mathbf{I}-\mathbf{A}_{2}\left(\mathbf{A}_{2}^{T} \Sigma^{-1} \mathbf{A}_{2}\right)^{-1} \mathbf{A}_{2}^{T} \Sigma^{-1}=\mathbf{I}-\mathbf{A}_{2} \mathbf{N}_{22}^{-1} \mathbf{A}_{2}^{T} \Sigma^{-1}$. It can be proved that the matrix $\mathbf{R}_{1}$ is idempotent and $\Sigma^{-1} \mathbf{R}_{1}$ is symmetric, see [25].

$$
\begin{aligned}
\mathbf{R}_{1} \mathbf{R}_{1} & =\mathbf{R}_{1}, \\
\boldsymbol{\Sigma}^{-1} \mathbf{R}_{1} & =\mathbf{R}_{1}^{T} \boldsymbol{\Sigma}^{-1} .
\end{aligned}
$$

It was proved that the estimator of $\widehat{\mathbf{x}}_{\mathrm{vp}, G}$ and its covariance matrix $\mathbf{D}\left(\widehat{\mathbf{x}}_{\mathrm{vp}, G}\right)$ can be written as

$$
\left\{\begin{array}{l}
\widehat{\mathbf{x}}_{\mathrm{vp}, G}=\mathbf{M}_{11}^{-1} \mathbf{A}_{1}^{T} \mathbf{R}_{1}^{T} \boldsymbol{\Sigma}^{-1} \mathbf{l}, \\
\mathbf{M}_{11}=\mathbf{A}_{1}^{T} \mathbf{R}_{1}^{T} \boldsymbol{\Sigma}^{-1} \mathbf{R}_{1} \mathbf{A}_{1}, \\
\mathbf{D}\left(\widehat{\mathbf{x}}_{\mathrm{vp}, G}\right)=\mathbf{M}_{11}^{-1},
\end{array}\right.
$$


which are equivalent to the solution of (26). Relying on (46), it can be proved that the LC and TC integrations are also equivalent in this case

$$
\mathbf{x}=\left[\begin{array}{lll}
\mathbf{x}_{\mathrm{avp}} & \mathbf{x}_{\mathrm{imu}} & \mathbf{x}_{\eta}
\end{array}\right]^{T} .
$$

With the state of (47), the LC measurement model is

$$
\begin{aligned}
\mathbf{H}_{k, \mathrm{LC}} & =\left[\begin{array}{llll}
0_{6 \times 3} & \mathbf{I}_{6} & 0_{6 \times 6} & \mathbf{T}_{\mathrm{vp}, \eta}
\end{array}\right], \\
\mathbf{z}_{k, \mathrm{LC}} & =\widehat{\mathbf{x}}_{\mathrm{vp}, G}, \\
\mathbf{R}_{k, \mathrm{LC}} & =\mathbf{M}_{11}^{-1} .
\end{aligned}
$$

Therefore, the LC KF solution is

$$
\left\{\begin{aligned}
\mathbf{P}_{k, \mathrm{LC}} & =\left(\mathbf{H}_{k, \mathrm{LC}}^{T} \mathbf{R}_{k, \mathrm{LC}}^{-1} \mathbf{H}_{k, \mathrm{LC}}+\overline{\mathbf{P}}_{k}^{-1}\right)^{-1} \\
& =\left(\mathbf{H}_{k, \mathrm{LC}}^{T} \mathbf{M}_{11} \mathbf{H}_{k, \mathrm{LC}}+\overline{\mathbf{P}}_{k}^{-1}\right)^{-1}, \\
\mathbf{K}_{k, \mathrm{LC}} & =\mathbf{P}_{k, \mathrm{LC}} \mathbf{H}_{k, \mathrm{LC}}^{T} \mathbf{R}_{k, \mathrm{LC}}^{-1}=\mathbf{P}_{k, \mathrm{LC}} \mathbf{H}_{k, \mathrm{LC}}^{T} \mathbf{M}_{11}, \\
\widehat{\mathbf{x}}_{k, \mathrm{LC}} & =\mathbf{K}_{k, \mathrm{LC}} \mathbf{z}_{k, \mathrm{LC}}=\mathbf{P}_{k, \mathrm{LC}} \mathbf{H}_{k, \mathrm{LC}}^{T} \mathbf{M}_{11} \widehat{\mathbf{x}}_{\mathrm{vp}, G} \\
& =\mathbf{P}_{k, \mathrm{LC}} \mathbf{H}_{k, \mathrm{LC}}^{T} \mathbf{A}_{1}^{T} \mathbf{R}_{1}^{T} \boldsymbol{\Sigma}^{-1} \mathbf{l} .
\end{aligned}\right.
$$

Accordingly, the TC measurement model is

$$
\begin{aligned}
\mathbf{H}_{k, \mathrm{TC}} & =\mathbf{R}_{1} \mathbf{A}_{1} \mathbf{H}_{k, \mathrm{TC}}, \\
\mathbf{z}_{k, \mathrm{TC}} & =\mathbf{l}, \\
\mathbf{R}_{k, \mathrm{TC}} & =\mathbf{\Sigma},
\end{aligned}
$$

Therefore, the TC KF solution is

$$
\left\{\begin{array}{l}
\mathbf{P}_{k, \mathrm{TC}}=\left(\mathbf{H}_{k, \mathrm{TC}}^{T} \mathbf{R}_{k, \mathrm{TC}}^{-1} \mathbf{H}_{k, \mathrm{TC}}+\overline{\mathbf{P}}_{k}^{-1}\right)^{-1} \\
=\left(\mathbf{H}_{k, \mathrm{LC}}^{T} M_{11} \mathbf{H}_{k, \mathrm{LC}}+\overline{\mathbf{P}}_{k}^{-1}\right)^{-1}=\mathbf{P}_{k, 1}, \\
\mathbf{K}_{k, \mathrm{TC}}=\mathbf{P}_{k, \mathrm{TC}} \mathbf{H}_{k, \mathrm{TC}}^{T} \mathbf{R}_{k, \mathrm{TC}}^{-1}=\mathbf{P}_{k, \mathrm{TC}} \mathbf{H}_{k, \mathrm{LC}}^{T} \mathbf{A}_{1}^{T} \mathbf{R}_{1}^{T} \boldsymbol{\Sigma}^{-1}, \\
\widehat{\mathbf{x}}_{k, \mathrm{TC}}=\mathbf{K}_{k, \mathrm{TC}} \mathbf{z}_{k, \mathrm{TC}}=\mathbf{P}_{k, \mathrm{TC}} \mathbf{H}_{k, \mathrm{LC}}^{T} \mathbf{A}_{1}^{T} \mathbf{R}_{1}^{T} \boldsymbol{\Sigma}^{-1} \mathbf{l}=\widehat{\mathbf{x}}_{k, \mathrm{LC}} .
\end{array}\right.
$$

(49) and (51) indicate that the equivalence between LC and TC is also satisfied in this case. It is noted that the equivalence between (36) and (49) and between (41) and (51) are not satisfied. In (36) and (41), the dynamic characteristic of GNSS receiver's clock bias and drift are considered in the KF predict model, while the corresponding dynamic information is ignored in (49) and (51).

\section{Measurement Models for GNSS Measurements at Multiple Epochs}

It is known that the LC integration requires less information exchange for integration but would be suspended if GNSS standalone solution failed. Comparably, the TC processor can improve the continuity and stability of the system, but it requires heavier information exchange and therefore complicates the computation. The above derivation concludes that the LC and TC algorithms are equivalent based on some implied conditions. Therefore, the most efficient procedure is to trigger the LC processor when the GNSS solution is provided and return to the TC processor when the available satellites are less than 4 . By this way, both the computation efficiency and the navigation reliability can be guaranteed.

GNSS precise positioning using carrier phase measurements implies a certain potential dynamic model, i.e., the ambiguity is constant when there is no cycle slip, and the residual ionospheric delay is temporarily stable. In this case, the solution is not "standalone" but time correlated. Therefore, the equivalence between LC and TC integrations should be rediscussed.

Firstly, the ambiguity fixing model is particularly discussed. For GNSS positioning procedure, the least-squares algorithm is applied at the $1^{\text {st }}$ epoch and the solution of position and velocity parameters is given as (46). It is noticed that (46) only makes senses when both pseudorange and carrier phase measurements are used, since the matrix $\mathbf{M}_{11}$ would be ranked deficient if only the carrier phase measurements are considered.

The single epoch least-squares solution of ambiguities is

$$
\left\{\begin{array}{l}
\widehat{\mathbf{x}}_{b}=\mathbf{M}_{22}^{-1} \mathbf{A}_{2}^{T} \mathbf{R}_{2}^{T} \boldsymbol{\Sigma}^{-1} \mathbf{l}, \\
\mathbf{M}_{22}=\mathbf{A}_{2}^{T} \mathbf{R}_{2}^{T} \boldsymbol{\Sigma}^{-1} \mathbf{R}_{2} \mathbf{A}_{2} \\
\mathbf{D}\left(\widehat{\mathbf{x}}_{b}\right)=\mathbf{M}_{22}^{-1}
\end{array}\right.
$$

with $\mathbf{R}_{2}=\mathbf{I}-\mathbf{A}_{1} \mathbf{N}_{11}^{-1} \mathbf{A}_{1}^{T} \Sigma^{-1}$. Without enough information, $\mathbf{M}_{22}$ is singular, so usually the norm equation is recorded as

$$
\left\{\begin{array}{l}
\mathbf{M}_{22} \widehat{\mathbf{x}}_{b}=\mathbf{U}_{2}, \\
\mathbf{U}_{2}=\mathbf{A}_{2}^{T} \mathbf{R}_{2}^{T} \boldsymbol{\Sigma}^{-1} \mathbf{l} .
\end{array}\right.
$$

At current epoch $k$, the normal equation is accumulated as

$$
\left\{\begin{array}{l}
\widetilde{\mathbf{x}}_{b}=\mathbf{M}_{k}^{-1} \mathbf{U}_{k} \\
\mathbf{D}\left(\widetilde{\mathbf{x}}_{b}\right)=\mathbf{M}_{k}^{-1} \\
\mathbf{M}_{k}=\sum_{i=1}^{k} \mathbf{M}_{22, i} \\
\mathbf{U}_{k}=\sum_{i=1}^{k} \mathbf{U}_{2, i}
\end{array}\right.
$$

where $i$ and $k$ are the epoch indicators. Accordingly, the solution of $\mathbf{x}_{\mathrm{vp}, G}$ with accumulated ambiguity float solution is

$$
\begin{aligned}
\widetilde{\mathbf{x}}_{\mathrm{vp}, G}= & \mathbf{N}_{11}^{-1} \mathbf{A}_{1}^{T} \boldsymbol{\Sigma}^{-1}\left(\mathbf{l}-\mathbf{A}_{2} \widetilde{\mathbf{x}}_{b}\right)=\mathbf{N}_{11}^{-1} \mathbf{A}_{1}^{T} \boldsymbol{\Sigma}^{-1} \\
& \cdot\left[\left(\mathbf{I}-\mathbf{A}_{2} \mathbf{M}_{k}^{-1} \mathbf{A}_{2}^{T} \mathbf{R}_{2}^{T} \boldsymbol{\Sigma}^{-1}\right) \mathbf{l}-\mathbf{A}_{2} \mathbf{M}_{k}^{-1} \mathbf{U}_{k-1}\right]
\end{aligned}
$$

and the variance matrix is

$$
\mathbf{D}\left(\widetilde{\mathbf{x}}_{\mathrm{vp}, G}\right)=\mathbf{N}_{11}^{-1}+\mathbf{N}_{11}^{-1} \mathbf{N}_{12} \mathbf{M}_{k}^{-1} \mathbf{N}_{21} \mathbf{N}_{11}^{-1}
$$

Intrinsically, the observation function for solutions (54), (55), and (56) can be rewritten as 


$$
\begin{aligned}
& {\left[\begin{array}{c}
\mathbf{l}_{1} \\
\mathbf{l}_{2} \\
\vdots \\
\mathbf{l}_{k}
\end{array}\right]=\left[\begin{array}{cc}
0 & \mathbf{R}_{2,1} \mathbf{A}_{2,1} \\
0 & \mathbf{R}_{2,2} \mathbf{A}_{2,2} \\
\vdots & \vdots \\
\mathbf{A}_{1, k} & \mathbf{A}_{2, k}
\end{array}\right]\left[\begin{array}{c}
\mathbf{x}_{\mathrm{vp}, G} \\
\mathbf{x}_{b}
\end{array}\right]+\left[\begin{array}{c}
\boldsymbol{\varepsilon}_{1} \\
\boldsymbol{\varepsilon}_{2} \\
\vdots \\
\boldsymbol{\varepsilon}_{k}
\end{array}\right],} \\
& \mathbf{D}(\boldsymbol{\varepsilon})=\left[\begin{array}{llll}
\Sigma_{1} & & & \\
& \Sigma_{2} & & \\
& & \ddots & \\
& & & \Sigma_{k}
\end{array}\right]
\end{aligned}
$$

where the subscript " $k$ " is the epoch indicator.

The single epoch solution of GNSS position and velocity is given by (46), and the covariance between $\widehat{\mathbf{x}}_{v p, G}$ and $\widehat{\mathbf{x}}_{b}$ is

$$
\mathbf{D}\left(\widehat{\mathbf{x}}_{\mathrm{vp}, G}, \widehat{\mathbf{x}}_{b}\right)=-\mathbf{M}_{11}^{-1} \mathbf{N}_{12} \mathbf{N}_{22}^{-1}
$$

As presented in the previous section, it is concluded that the LC measurement mode with (46), (52), and (58) is equivalent to the TC measurement mode with (25). For these solutions, the ambiguity should be included in the integration state vector and its single epoch float solution should be used as the measurement update, so that the multiple epoch ambiguity information is reserved in the integration procedure. Otherwise, if only the position or/and velocity from GNSS standalone solution is involved in the integration procedure, the ambiguity cannot be fixed, since the ambiguity dynamic constraints are not reserved.

The LC procedure with states including ambiguities and measurement update from (54), (55), and (56) is equivalent to the TC procedure of using (57) as the measurement update. This mode uses the multiple epoch ambiguity solution twice, both in the integration dynamic and measurement model. Therefore, the integration solution is naturally different with that of using (46), (52), and (58).

The above derivation is easily extended to the case if the GNSS procedure contains another dynamic model. Generally, the GNSS solution with a dynamic model is given as

$$
\left\{\begin{array}{l}
\mathbf{x}_{G, 0}=\mathbf{x}_{G}+\mathbf{w} \\
\mathbf{l}=\mathbf{A} \mathbf{x}_{G}+\boldsymbol{\varepsilon}
\end{array}\right.
$$

where $\mathbf{w}$ is the state dynamic noise, and its variance is $\mathbf{D}(\mathbf{w})=\mathbf{D}\left(\mathbf{x}_{G, 0}\right)=\Sigma_{x}$; the measurement model is the same as (25). Supposing the solution of (59) is written as $\widetilde{\mathbf{x}}_{G}$ and $\mathbf{D}\left(\widetilde{\mathbf{x}}_{G}\right)$, therefore, the LC procedure of using $\widetilde{\mathbf{x}}_{G}$ and $\mathbf{D}\left(\widetilde{\mathbf{x}}_{G}\right)$ as the measurement update is equivalent to the TC procedure of using measurement update, as

$$
\left[\begin{array}{c}
\mathbf{x}_{G, 0} \\
\mathbf{l}
\end{array}\right]=\left[\begin{array}{c}
\mathbf{I} \\
\mathbf{A}
\end{array}\right] \mathbf{T}_{g, i} \mathbf{x}+\left[\begin{array}{l}
\mathbf{w} \\
\boldsymbol{\varepsilon}
\end{array}\right] .
$$

The corresponding dynamic model containing IMU information is

$$
\begin{aligned}
\mathbf{x} & =\overline{\mathbf{x}}_{1}, \\
\mathbf{D}\left(\overline{\mathbf{x}}_{1}\right) & =\overline{\mathbf{P}}_{1} .
\end{aligned}
$$

Supposing

$$
\begin{aligned}
\overline{\mathbf{x}}_{2} & =\mathbf{T}_{i, g} \mathbf{x}_{G, 0}, \\
\mathbf{D}\left(\overline{\mathbf{x}}_{2}\right) & =\overline{\mathbf{P}}_{2}=\mathbf{T}_{i, g} \Sigma_{x} \mathbf{T}_{i, g}^{T}
\end{aligned}
$$

Integrated estimation with (60) and (61) is also equivalent to the following estimation:

$$
\left\{\begin{array}{l}
\overline{\mathbf{x}}=\overline{\mathbf{P}}\left(\overline{\mathbf{P}}_{1}^{-1} \overline{\mathbf{x}}_{1}+\overline{\mathbf{P}}_{2}^{-1} \overline{\mathbf{x}}_{2}\right), \\
\overline{\mathbf{P}}=\left(\overline{\mathbf{P}}_{1}^{-1}+\overline{\mathbf{P}}_{2}^{-1}\right)^{-1} \\
\mathbf{l}=\mathbf{A T}_{g, i} \mathbf{x}+\boldsymbol{\varepsilon}
\end{array}\right.
$$

(63) means that the GNSS state model (59) is finally added to the integration state model (61). This is the reason that federal KF requires information allocation for the dynamic model among each subfilter [26, 27]. The same dynamic model used in each subfilter indicates that the dynamic variance in the main filter is reduced by a factor of $1 / n$, where $n$ is the number of the subfilter.

The models used for GNSS/INS integration are summarized in Table 2, with the equivalent LC and TC measurement models presented at the last two columns. If the GNSS solution is unavailable, the normal equation is used as the measurement model for integration. It should be noted that the equivalence is satisfied within each model and shows discrepancies among different models, i.e., models 1 and 2 are different due to the dynamic of $\mathbf{x}_{t}$. If $\mathbf{x}_{t}$ is modelled as random noise and the dynamic noise tends to infinite, models 1 and 2 would be approximately equivalent. Models 3,4 , and 5 are for RTK solution. With the same dynamic model for $\mathbf{x}_{b}$, the integration solution of models 3 and 4 would be similar, while model 5 cannot fix the ambiguity.

\section{Experiment and Analysis}

The above derivation proves that the LC and TC integrations are naturally equivalent when the same information is used. Usually, the measurement stochastic model of the LC integration is empirically determined rather than derived from the GNSS procedure. This is the reason that caused the discrepancies. A flight test dataset is used to verify the discrepancies among different models. The flight test, with trajectory shown in Figure 1, was conducted on October 17, 2011, from Bankstown Airport, Sydney to Cooma Airport, which is in the southern part of New South Wales, Australia. The GNSS data was collected by a Leica dual-frequency receiver with a sample rate of $10 \mathrm{~Hz}$. The cutoff elevation angle was $10^{\circ}$ and the number of visible GPS satellites was nine throughout the experimental period. The INS data collected the KVH's IMU embedded in the SPAN-CPT with the specification listed in Table 3, and the measurement rate was set to $100 \mathrm{~Hz}$.

5.1. Comparisons of Using GNSS Pseudoranges. Firstly, the solutions with GNSS SPP processor are compared and 15 states with position, velocity, attitude, acceleration, and gyroscope errors are selected. State model 2 in Table 2 is 
TABLE 2: Different state models for the GNSS/INS integration ( $\widehat{\mathbf{x}}$ means standalone solution; $\widetilde{\mathbf{x}}$ means KF solution with state prediction).

\begin{tabular}{|c|c|c|c|c|c|}
\hline No. & GNSS solution & Integration state includes & GNSS states & GNSS observation function TC measurements & $\begin{array}{c}\text { GNSS solution LC } \\
\text { measurements }\end{array}$ \\
\hline 1 & SPP & $\left(\begin{array}{ll}\mathbf{x}_{v p}^{T} & \mathbf{x}_{t}^{T}\end{array}\right)^{T}$ & $\mathbf{x}^{T}=\left(\begin{array}{ll}\mathbf{x}_{\mathrm{vp}, G}^{T} & \mathbf{x}_{t}^{T}\end{array}\right)^{T}$ & $\mathbf{l}=\mathbf{A}_{1} \mathbf{x}_{\mathrm{vp}, G}+\mathbf{A}_{2} \mathbf{x}_{t}+\varepsilon$ & $\widehat{\mathbf{x}}, \mathbf{D}(\widehat{\mathbf{x}})$ \\
\hline 2 & SPP/DGPS & $\mathbf{x}_{\mathrm{vp}}^{T}$ & $\mathbf{x}_{\mathrm{vp}, G}^{T}$ & $\mathbf{l}=\mathbf{R}_{1} \mathbf{A}_{1} \mathbf{x}_{\mathrm{vp}, G}+\varepsilon$ & $\widehat{\mathbf{x}}_{\mathrm{vp}, G}, \mathbf{D}\left(\widehat{\mathbf{x}}_{\mathrm{vp}, G}\right)$ \\
\hline 3 & RTK & $\left(\begin{array}{ll}\mathbf{x}_{\mathrm{vp}}^{T} & \mathbf{x}_{b}^{T}\end{array}\right)^{T}$ & $\mathbf{x}^{T}=\left(\begin{array}{ll}\mathbf{x}_{\mathrm{vp}, G}^{T} & \mathbf{x}_{b}^{T}\end{array}\right)^{T}$ & $\mathbf{l}=\mathbf{A}_{1} \mathbf{x}_{\mathrm{vp}, G}+\mathbf{A}_{2} \mathbf{x}_{b}+\varepsilon$ & $\widehat{\mathbf{x}}, \mathbf{D}(\widehat{\mathbf{x}})$ \\
\hline 4 & RTK & $\mathbf{x}_{\mathrm{vp}}^{T}$ & $\mathbf{x}^{T}=\left(\begin{array}{ll}\mathbf{x}_{\mathrm{vp}, G}^{T} & \mathbf{x}_{b}^{T}\end{array}\right)^{T}$ & $\left\{\begin{array}{l}\dot{x}_{b}=0 \\
\mathbf{l}=\mathbf{A}_{1} \mathbf{x}_{\mathrm{vp}, G}+\mathbf{A}_{2} \mathbf{x}_{b}+\varepsilon\end{array}\right.$ & $\widetilde{\mathbf{x}}_{\mathrm{vp}, G}, \mathbf{D}\left(\widetilde{\mathbf{x}}_{\mathrm{vp}, G}\right)$ \\
\hline 5 & RTK & $\mathbf{x}_{\mathrm{vp}}^{T}$ & $\mathbf{x}_{\mathrm{vp}, G}^{T}$ & $\mathbf{l}=\mathbf{R}_{1} \mathbf{A}_{1} \mathbf{x}_{\mathrm{vp}, G}+\varepsilon$ & $\widehat{\mathbf{x}}_{\mathrm{vp}, G}, \mathbf{D}\left(\widehat{\mathbf{x}}_{\mathrm{vp}, G}\right)$ \\
\hline
\end{tabular}

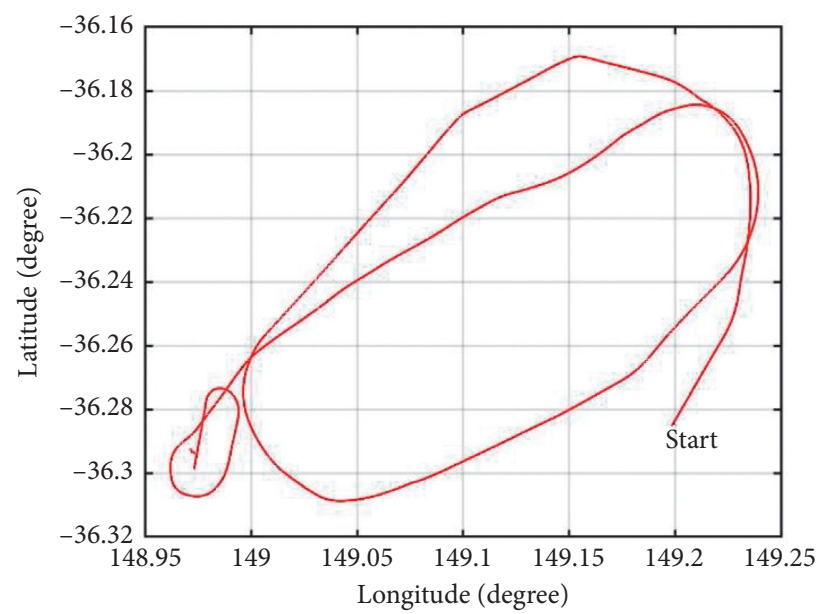

Figure 1: Trajectory of the flight test.

TABLE 3: Technical parameters of the IMU.

Technical parameters DQI of KVH IMU

Accelerometer bias

TABLE 4: Four measurement models for integration with GNSS pseudorange measurements (unit: $\mathrm{m}$ for positions and pseudoranges and $\mathrm{m} / \mathrm{s}$ for velocities).

\begin{tabular}{|c|c|c|c|}
\hline Model & Schemes & Integration measurements & Measurement variance for integration \\
\hline A & Loosely & Position and velocity & $\mathbf{R}=\operatorname{diag}\left[\begin{array}{llllll}4 & 4 & 16 & 0.04 & 0.04 & 16\end{array}\right]$ \\
\hline $\mathrm{B}$ & Loosely & Position and velocity & $\mathbf{R}=\mathbf{D}\left(\widehat{\mathbf{x}}_{G}\right)=\left(\begin{array}{lll}\mathbf{A}^{T} \Sigma^{-1} & \mathbf{A}\end{array}\right)^{-1}$ \\
\hline $\mathrm{C}$ & Loosely & Position and velocity & $\mathbf{R}=\operatorname{diag}\left(\mathbf{D}\left(\widehat{\mathbf{x}}_{G}\right)\right)=\operatorname{diag}\left(\left(\begin{array}{lll}\mathbf{A}^{T} \Sigma^{-1} & \mathbf{A}\end{array}\right)^{-1}\right)$ \\
\hline $\mathrm{D}$ & Tightly & Corrected pseudoranges & $\mathbf{R}=\mathbf{D}(\varepsilon)=\Sigma$ \\
\hline
\end{tabular}

select as demonstration, and four different measurement models listed in Table 4 are compared.

The integration solution from the Inertial Explorer software is used as the reference solution and the position, velocity, and attitude errors of the four models are shown in Figures 2-4.

Figure 2 shows that the horizontal position errors are all within 2 meters and vertical position errors are around 5 meters. The position results show some systematic biases, about 1 meter on horizontal and 2 meters on vertical direction. Generally, the solutions for models B, C, and D are quite close and much more stable than that of model A. Theoretical derivation proves that the solutions of model B and D are equivalent. The zoom-in plots in each panel of Figure 2 show that the results of models B and D are same in millimeter level and their discrepancies with model C are also small, usually around several centimeters. 

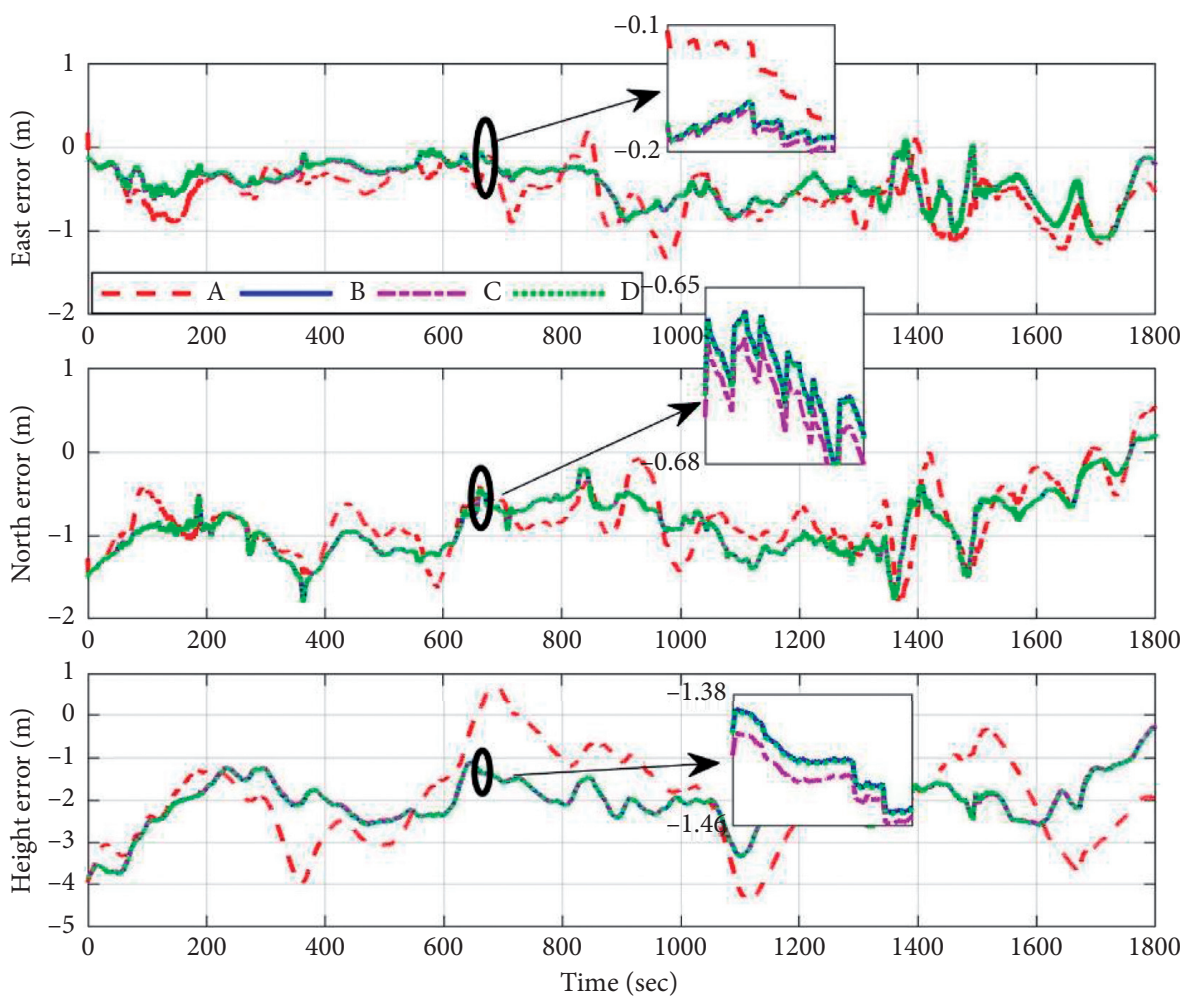

FIgURE 2: Position errors of using GNSS pseudoranges for integration when different models are used (A,loosely coupled with empirical variances; B, loosely coupled with variances from GNSS SPP; C, loosely coupled with variance from GNSS SPP neglecting the covariance; D, tightly coupled with GNSS pseudoranges' variances).
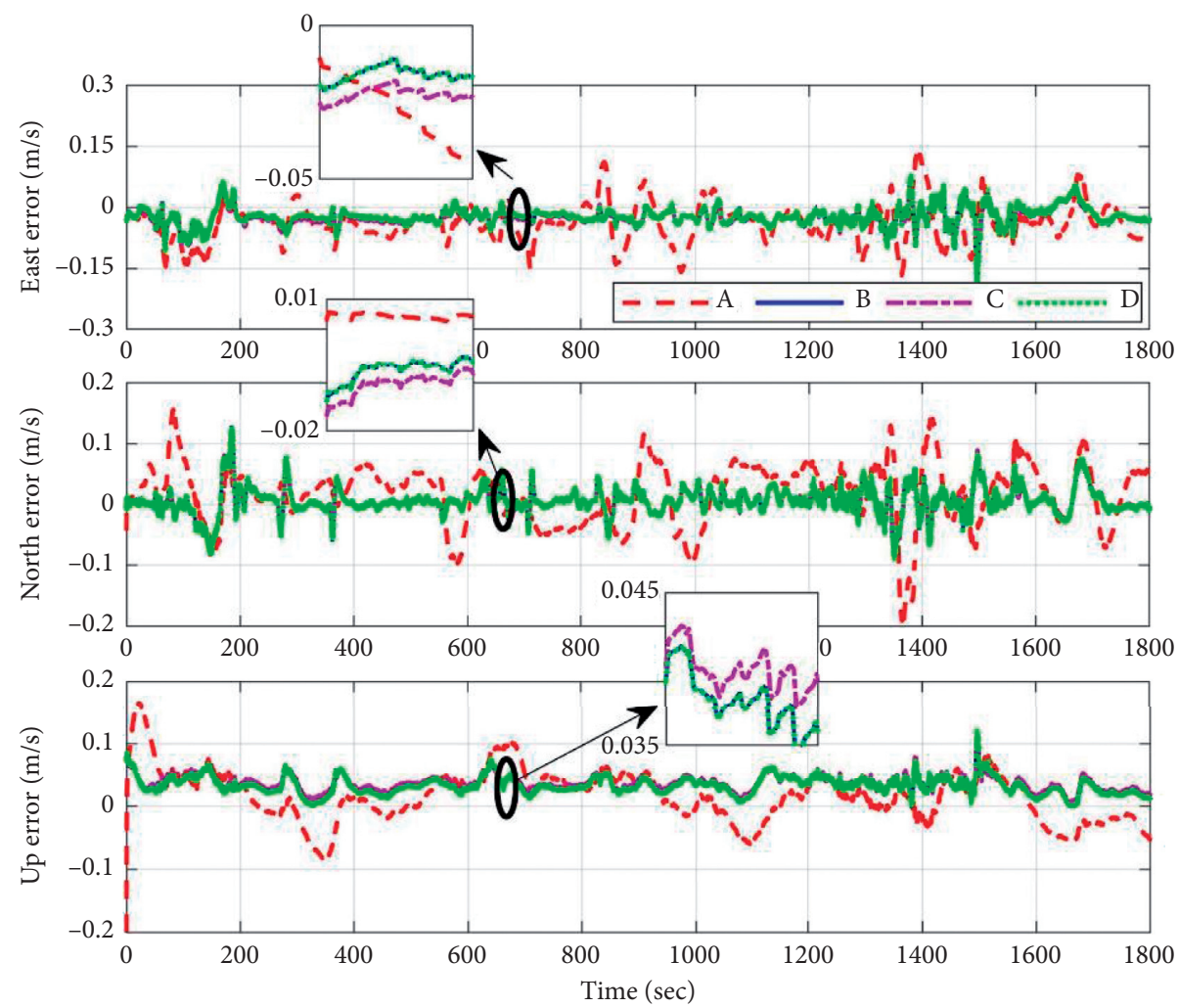

FIGURE 3: Velocity errors of using GNSS pseudoranges for integration when different models are used (A, loosely coupled with empirical variances; B, loosely coupled with variances from GNSS SPP; C, loosely coupled with variance from GNSS SPP neglecting the covariance; D, tightly coupled with GNSS pseudoranges' variances). 

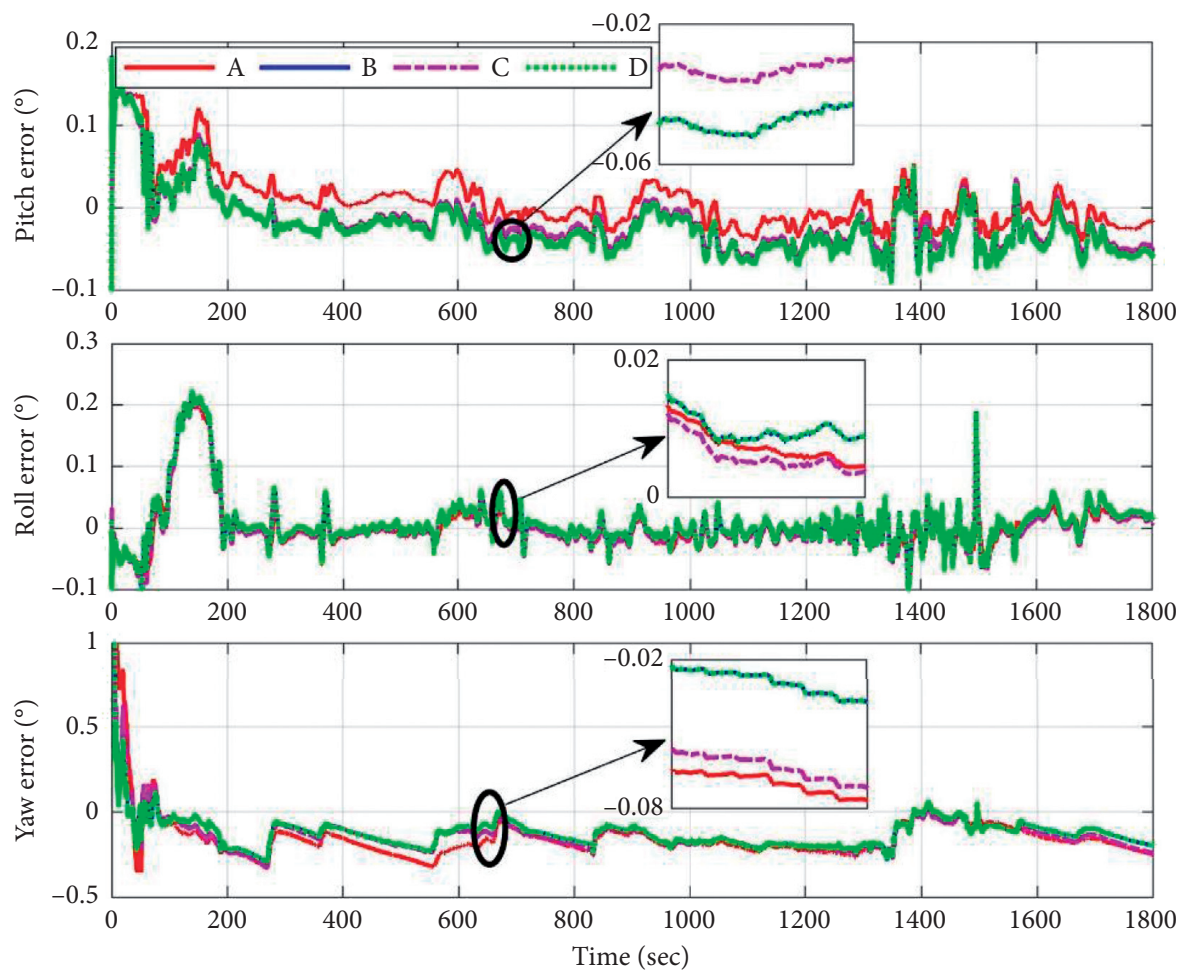

FIgURE 4: Attitude errors of using GNSS pseudoranges for integration when different models are used. (A, loosely coupled with empirical variances; B, loosely coupled with variances from GNSS SPP; C, loosely coupled with variance from GNSS SPP neglecting the covariance; D, tightly coupled with GNSS pseudoranges' variances).

Figure 3 shows that velocity errors on the three directions are within $0.3 \mathrm{~m} / \mathrm{s}$. Similarly, the velocity estimation errors of models B, C, and D are obviously more stable and smaller than that of model A. The zoom-in plots further prove that the results of $\mathrm{B}$ and $\mathrm{D}$ are always close and the differences with model $\mathrm{C}$ are within $1 \mathrm{~cm} / \mathrm{s}$.

Figure 4 shows tthe attitude errors of the four models, within 0.2 degrees on pitch and roll and 0.5 degrees on yaw after converging. The top panel shows that pitch errors of model A have significant biases with that of models B, C, and D. Middle and bottom panels show that biases on roll and yaw between different models are smaller.

Generally, results in Figures 2-4 show that the LC and TC integrations provide equivalent solutions once the LC measurement variances are estimated by the GNSS processor, which is a function of the TC measurement variances. Also, it shows that if empirical constant variances were used for LC measurement update, the solution accuracy and stability would degrade.

Table 5 lists the mean and standard deviation (STD) values of the position, velocity, and attitude errors by using the four models. Generally, performance of model A is the worst, and performances of models $\mathrm{B}$ and $\mathrm{D}$ are almost the same and little better than model C. In terms of absolute mean values, the discrepancies among the four models are generally small, except for the yaw errors, where errors of models B, C and D are much smaller than those of model A. The differences on STD values are much more significant. For position solutions, improvements of models B, C, ND D compared with model A are around 15\% on horizontal and $40 \%$ on vertical direction. The corresponding improvements on velocity solutions are much larger, around $51 \%$ on horizontal and $66 \%$ on vertical direction. The improvements on pitch and roll solutions are little, but quite significant on the yaw axis, around 25\%. Results of models B and D are always the same on the third decimal place. Model $\mathrm{C}$ also shows little differences with models $\mathrm{B}$ and $\mathrm{D}$, which indicates that the covariance of position and velocity can be neglected in some cases when the correlation is insignificant.

5.2. Comparison of Using GNSS Pseudoranges and Carrier Phases. In this subsection, the integration solutions with the precise GNSS RTK processor are compared and 18 states with three lever arm error states are considered. The state model 5 in Table 2 is selected for demonstration and the four measurement models for integration in Table 4 are compared with the measurement variances of model $\mathrm{A}$ as $\mathbf{R}=\operatorname{diag}\left[\begin{array}{llllll}0.01 & 0.01 & 0.16 & 0.04 & 0.04 & 0.16\end{array}\right]$, since the GNSS processor provides RTK solutions. The corresponding position, velocity, and attitude errors of the four models are shown in Figures 5-7.

Figure 5 shows that the position errors are all within 0.4 meters and the horizontal accuracies are obviously higher than the vertical accuracy. Generally, similar to the results of using pseudoranges, the errors of model A fluctuate more severely compared with other three models. The zoom-in figures show that the discrepancies among models B, C, and $\mathrm{D}$ are always small at millimeter level for horizontal and 
TABLE 5: Mean and STD values of position, velocity, and attitude errors of using GNSS pseudoranges for integration when different integrations are used.

\begin{tabular}{|c|c|c|c|c|c|c|c|c|}
\hline & \multicolumn{4}{|c|}{ Mean } & \multicolumn{4}{|c|}{ STD } \\
\hline & A & $\mathrm{B}$ & $\mathrm{C}$ & $\mathrm{D}$ & A & $\mathrm{B}$ & $\mathrm{C}$ & $\mathrm{D}$ \\
\hline $\mathrm{E}(\mathrm{m})$ & 0.5805 & 0.4438 & 0.4526 & 0.4436 & 0.2843 & 0.2394 & 0.2370 & 0.2395 \\
\hline $\mathrm{N}(\mathrm{m})$ & 0.8165 & 0.8680 & 0.8676 & 0.8681 & 0.4351 & 0.3910 & 0.3905 & 0.3910 \\
\hline $\mathrm{U}(\mathrm{m})$ & 2.0099 & 2.0454 & 2.0333 & 2.0456 & 1.0576 & 0.6215 & 0.6237 & 0.6215 \\
\hline $\mathrm{Ve}(\mathrm{m} / \mathrm{s})$ & 0.0512 & 0.0266 & 0.0289 & 0.0266 & 0.0476 & 0.0229 & 0.0221 & 0.0229 \\
\hline $\operatorname{Vn}(\mathrm{m} / \mathrm{s})$ & 0.0440 & 0.0156 & 0.0152 & 0.0156 & 0.0503 & 0.0223 & 0.0217 & 0.0223 \\
\hline $\mathrm{Vu}(\mathrm{m} / \mathrm{s})$ & 0.0352 & 0.0330 & 0.0360 & 0.0330 & 0.0416 & 0.0140 & 0.0122 & 0.0140 \\
\hline Pitch (degree) & 0.0242 & 0.0356 & 0.0312 & 0.0356 & 0.0304 & 0.0370 & 0.0351 & 0.0370 \\
\hline Roll (degree) & 0.0231 & 0.0244 & 0.0239 & 0.0244 & 0.0417 & 0.0430 & 0.0421 & 0.0430 \\
\hline Yaw (degree) & 0.1822 & 0.1371 & 0.1549 & 0.1371 & 0.1635 & 0.1160 & 0.1267 & 0.1167 \\
\hline
\end{tabular}

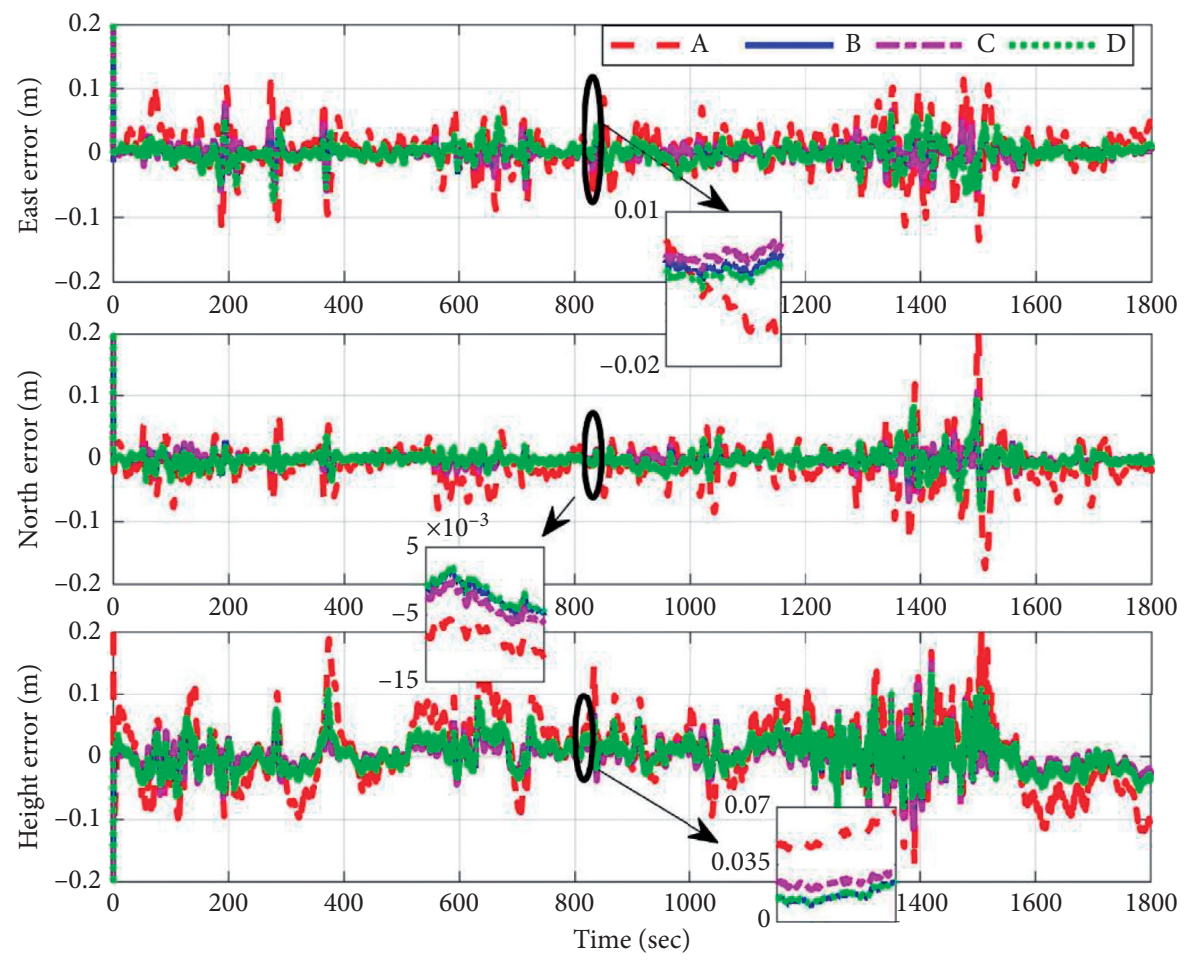

FIGURE 5: Position errors of using GNSS pseudoranges and carrier phases for integration when different models are used (A, loosely coupled with empirical variances; B, loosely coupled with variances from GNSS SPP; C, loosely coupled with variance from GNSS SPP neglecting the covariance; D, tightly coupled with GNSS pseudoranges' variances).

centimeter level for vertical position errors. Figure 6 shows the velocity errors of using the four models. The velocity error discrepancies among models are not as obvious as that of the position errors, and the results of models $\mathrm{B}$ and $\mathrm{D}$ are still close. Figure 7 shows the corresponding attitude errors. The top panel shows that the pitch errors of model A are even smaller than that of other three models, especially during the 200 1200 second. This may be caused by the uncertainty of the carrier phase variances. The middle panel shows that the roll errors of the four models are quite close, but the errors of model A seem slightly smaller. The bottom panel shows that the yaw solutions of models B, C, and D are obviously higher than that of model $\mathrm{A}$, especially during the first 700 seconds.
Generally, Figures 2-7 reveal that models B and D can provide equivalent solutions, while their differences with model C is little but large with model A. In most cases, using the estimated variances from the GNSS processor as the integration measurements' variances can improve the accuracy and stability of the system.

The corresponding absolute mean and STD values of the position, velocity, and attitude errors are listed in Table 6. Generally, results of models B and D are mostly close. The STD values for position errors show that the estimation precision of the four models are comparable, while the absolute mean values of models $\mathrm{B}, \mathrm{C}$, and $\mathrm{D}$ are much better than that of model $\mathrm{A}$, indicating higher estimation accuracy. The velocity estimation accuracy and 

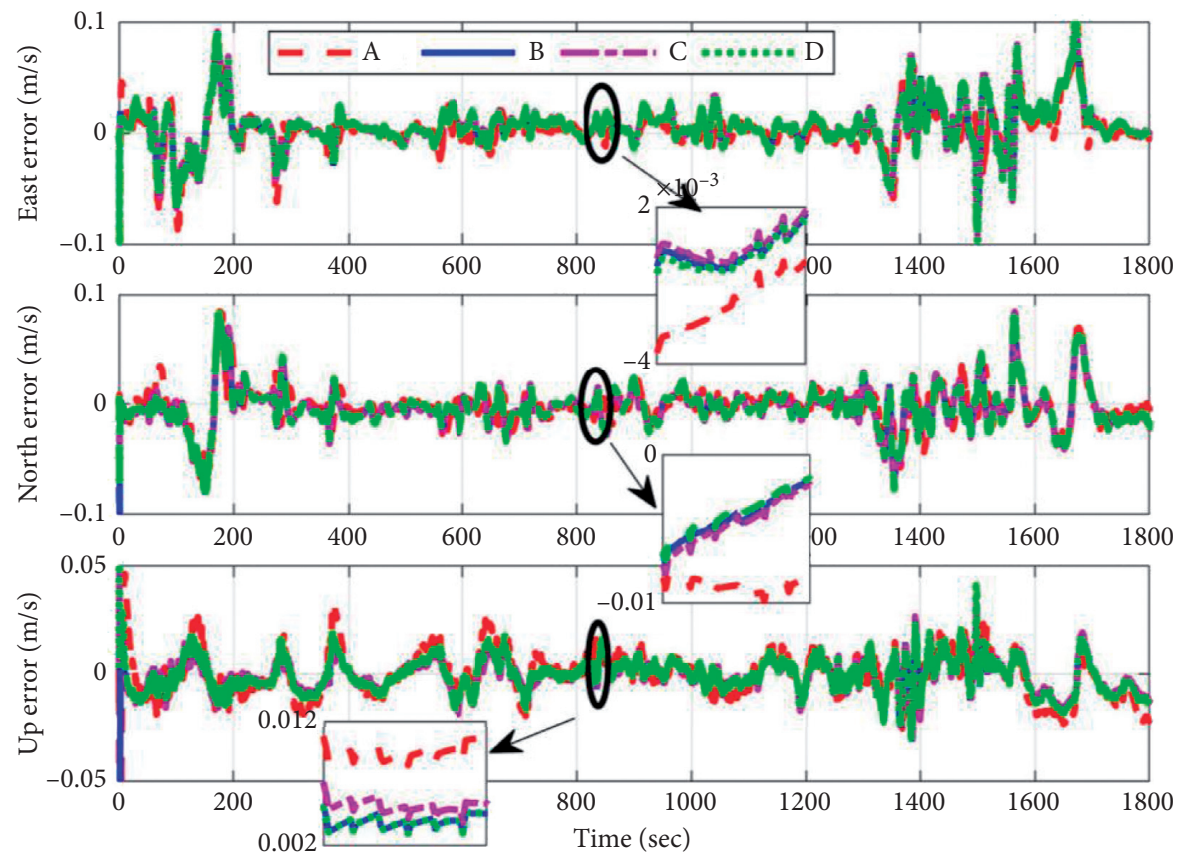

FIGURE 6: Velocity errors of using GNSS pseudoranges and carrier phases for integration when different models are used (A, loosely coupled with empirical variances; B, loosely coupled with variances from GNSS SPP; C, loosely coupled with variance from GNSS SPP neglecting the covariance; D, tightly coupled with GNSS pseudoranges' variances).
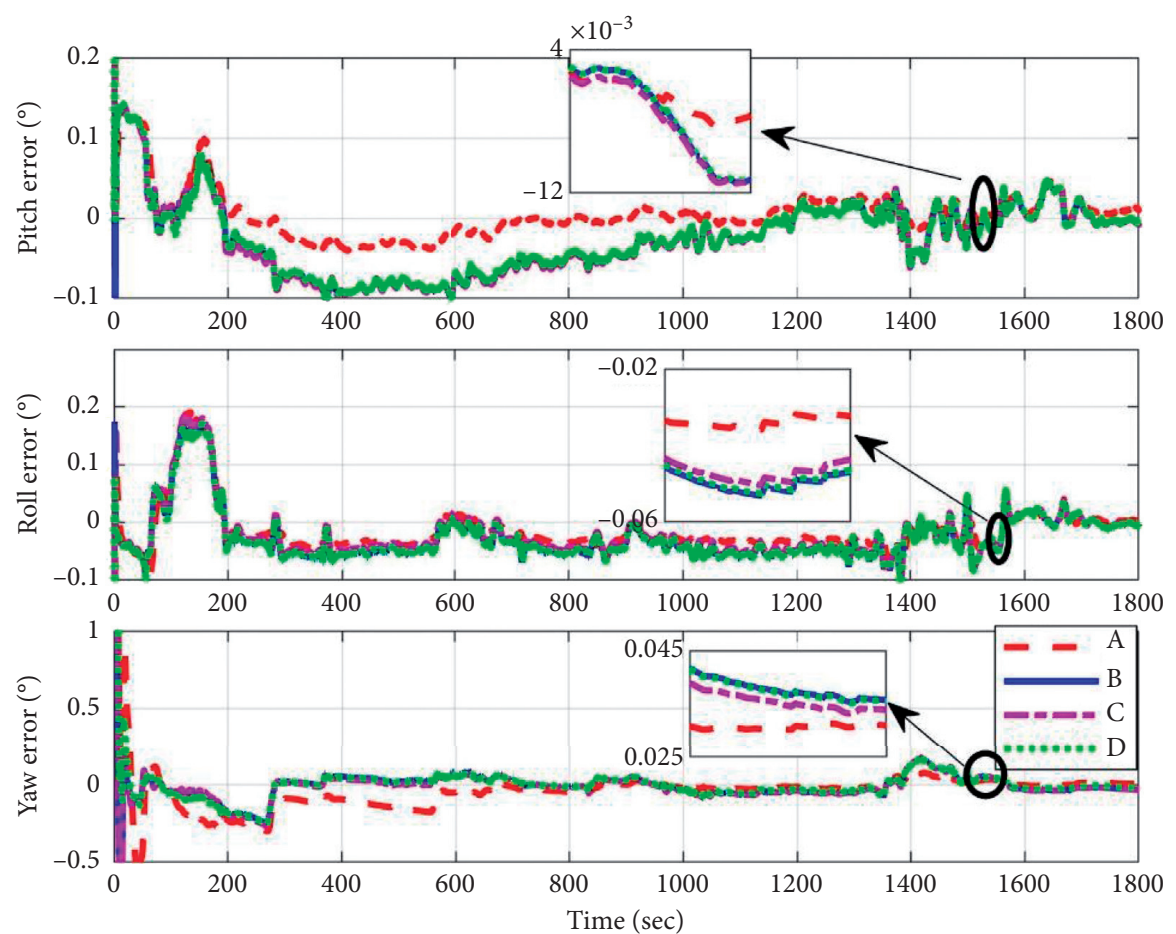

FIGURE 7: Attitude errors of using GNSS pseudoranges and carrier phases for integration when different models are used (A, loosely coupled with empirical variances; $B$, loosely coupled with variances from GNSS SPP; C, loosely coupled with variance from GNSS SPP neglecting the covariance; D, tightly coupled with GNSS pseudoranges' variances).

precision of the four models are comparable. For the pitch and roll estimation, the precisions of the four models are similar, while the accuracy of model A is much higher than that of other three models. Much more significant differences between model A and other three models appear on the yaw estimation. Compared with model A, both the 
TABLE 6: Mean and STD values of position, velocity, and attitude errors of using GNSS pseudoranges for integration when different integrations are used.

\begin{tabular}{lcccccrrr}
\hline & & \multicolumn{2}{c}{ Mean } & & & STD & \\
& A & B & C & D & A & B & C \\
\hline$E(\mathrm{~m})$ & 0.0259 & 0.0095 & 0.0120 & 0.0094 & 0.0338 & 0.0403 & 0.0558 & 0.0390 \\
$N(\mathrm{~m})$ & 0.0243 & 0.0082 & 0.0104 & 0.0081 & 0.0322 & 0.0411 & 0.0566 & 0.0383 \\
$U(\mathrm{~m})$ & 0.0470 & 0.0191 & 0.0104 & 0.0192 & 0.0575 & 0.0420 & 0.0477 & 0.0430 \\
$V e(\mathrm{~m} / \mathrm{s})$ & 0.0119 & 0.0141 & 0.0139 & 0.0140 & 0.0194 & 0.0198 & 0.0198 & 0.0198 \\
$V n(\mathrm{~m} / \mathrm{s})$ & 0.0113 & 0.0127 & 0.0125 & 0.0126 & 0.0176 & 0.0186 & 0.0184 & 0.0185 \\
$V u(\mathrm{~m} / \mathrm{s})$ & 0.0089 & 0.0066 & 0.0061 & 0.0065 & 0.0134 & 0.0078 & 0.0220 & 0.0084 \\
Pitch (degree) & 0.0201 & 0.0407 & 0.0410 & 0.0394 & 0.0314 & 0.0466 & 0.0457 & 0.0468 \\
Roll (degree) & 0.0315 & 0.0415 & 0.0375 & 0.0403 & 0.0423 & 0.0445 & 0.0432 \\
Yaw (degree) & 0.0764 & 0.0542 & 0.0558 & 0.0542 & 0.1441 & 0.1075 & 0.1133 & 0.10444 \\
\hline
\end{tabular}

estimation accuracy and precision of models B, C, and D are improved around $25 \%$.

\section{Concluding Remarks}

This paper proves the equivalence of the GNSS/INS loosely and tightly coupled integration based on four conditions: (1) in TC processor, the INS time update is used as the initial value for the linearization of the GNSS observation function; (2) the GNSS processor provides a standalone solution, which means no dynamic model was used for the solution; (3) if dynamic constraints are considered in the GNSS solution for the LC processor, the same dynamic information should be added to the dynamic model of the TC processor; (4) the covariance of the LC measurements should be derived from the GNSS solution instead of conventional empirical values. Upon this, different state models for integration are given to illustrate the natural differences between different filtering. This indicates that once the same measurement and predict information are used, the integration would be equivalent no matter what kind of coupling schemes are used. Therefore, to reserve the estimation efficiency, accuracy, and stability, the optimal procedure is to use the LC processor when the GNSS standalone solution is available and to use the TC processor when GNSS measurements are not enough. Alternatively, one can always use the norm equation from the GNSS processor for the integration measurement update, so that a unified model can be implemented in a practical processor.

\section{Data Availability}

The GNSS and IMU raw data used to support the findings of this study are available from the corresponding author upon request.

\section{Conflicts of Interest}

The authors declare no conflicts of interest.

\section{Acknowledgments}

This research was supported by the State Key Laboratory of Satellite Navigation System and Equipment Technology
(CEPNT-2017KF-10), the National Natural Science Foundation of China (41504022, 41474017, and 41730102), and the National Key R\&D Program of China (2017YFA0603102).

\section{References}

[1] S. Godha and M. E. Cannon, "GPS/MEMS INS integrated system for navigation in urban areas," GPS Solutions, vol. 11, no. 3, pp. 193-203, 2007.

[2] F. Caron, E. Duflos, D. Pomorski, and P. Vanheeghe, "GPS/ IMU data fusion using multisensor Kalman filtering: introduction of contextual aspects," Information Fusion, vol. 7, no. 2, pp. 221-230, 2006.

[3] S. Gao, Y. Zhong, X. Zhang, and B. Shirinzadeh, "Multi-sensor optimal data fusion for INS/GPS/SAR integrated navigation system," Aerospace Science and Technology, vol. 13, no. 4-5, pp. 232-237, 2009.

[4] L. Yang, Y. Li, Y. Wu, and C. Rizos, "An enhanced MEMSINS/GNSS integrated system with fault detection and exclusion capability for land vehicle navigation in urban areas," GPS Solutions, vol. 18, no. 4, pp. 593-603, 2014.

[5] P. Groves, Principles of GNSS, Inertial, and Multisensor Integrated Navigation Systems, Artech House, Norwood, MA, USA, 2nd edition, 2015.

[6] Z. Zhou, Y. Li, J. Liu, and G. Li, "Equality constrained robust measurement fusion for adaptive kalman-filter-based heterogeneous multi-sensor navigation," IEEE Transactions on Aerospace and Electronic Systems, vol. 49, no. 4, pp. 21462157, 2013.

[7] K. Chiang, H. Hou, X. Niu, and N. El-Sheimy, "Improving the positioning accuracy of DGPS/MEMS IMU integrated systems utilizing cascade de-noising algorithm," in Proceedings of the 17th International Technical Meeting of the Satellite Division of the Institute of Navigation (ION GNSS 2004), pp. 809-818, Long Beach, CA, USA, September 2004.

[8] J. A. Farrell, T. D. Givargis, and M. J. Barth, "Real-time differential carrier phase GPS-aided INS," IEEE Transactions on Control Systems Technology, vol. 8, no. 4, pp. 709-721, 2000.

[9] J. A. Farrell, H.-S. Tan, and Y. Yang, "Carrier phase GPS-aided INS-based vehicle lateral control," Journal of Dynamic Systems, Measurement, and Control, vol. 125, no. 3, pp. 339-353, 2003.

[10] Y. Zhang and Y. Gao, "Performance analysis of a tightly coupled kalman filter for the integration of un-differenced GPS and inertial data," in Proceedings of the 2005 National Technical Meeting of the Institute of Navigation, pp. 270-275, San Diego, CA, USA, January 2005. 
[11] Z. Zhang, B. Li, and Y. Shen, "Comparison and analysis of unmodelled errors in GPS and BeiDou signals," Geodesy and Geodynamics, vol. 8, no. 1, pp. 41-48, 2017.

[12] Z. Zhou, Y. Li, J. Zhang, and C. Rizos, "Integrated navigation system for a low-cost quadrotor aerial vehicle in the presence of rotors influences," Journal of Surveying Engineering, vol. 143, no. 1, 2017.

[13] Z. Zhou, J. Wu, Y. Li, C. Fu, and H. Fourati, "Critical issues on kalman filter with colored and correlated system noises," Asian Journal of Control, vol. 19, no. 6, pp. 1905-1919, 2017.

[14] D. Gu and N. El-Sheimy, "Heading accuracy improvement of MEMS IMU/DGPS integrated navigation system for land vehicle," in Proceedings of the 2008 IEEE/ION Position, Location and Navigation Symposium, pp. 1292-1296, Monterey, CA, USA, May 2008.

[15] M. Rabbou and A. El-Rabbany, "Tightly coupled integration of GPS precise point positioning and MEMS-based inertial systems," GPS Solutions, vol. 19, no. 4, pp. 601-609, 2015.

[16] G. Roesler and H. Martell, "Tightly coupled processing of precise point position (PPP) and INS data," in Proceedings of the 22nd International Meeting of the Satellite Division of the Institute of Navigation, pp. 1898-1905, Savannah, GA, USA, 2009.

[17] Z. Gao, H. Zhang, M. Ge et al., "Tightly coupled integration of ionosphere-constrained precise point positioning and inertial navigation systems," Sensors, vol. 15, no. 3, pp. 5783-5802, 2015.

[18] C. Eling, P. Zeimetz, and H. Kuhlmann, "Development of an instantaneous GNSS/MEMS attitude determination system," GPS Solutions, vol. 17, no. 1, pp. 129-138, 2013.

[19] G. Arshal, "Error equations of inertial navigation," Journal of Guidance Control and Dynamics, vol. 10, no. 4, pp. 351-358, 1987.

[20] D. Goshenmeskin and I. Baritzhack, "Unified approach to inertial navigation system error modeling," Journal of Guidance Control \& Dynamics, vol. 1, no. 3, pp. 472-480, 1992.

[21] G. Hu, B. Gao, Y. Zhong, and C. Gu, "Unscented kalman filter with process noise covariance estimation for vehicular INS/ GPS integration system," Information Fusion, vol. 64, pp. 194-204, 2020.

[22] G. Hu, L. Ni, B. Gao, X. Zhu, W. Wang, and Y. Zhong, "Model predictive based unscented kalman filter for hypersonic vehicle navigation with INS/GNSS integration," IEEE Access, vol. 8, pp. 4814-4823, 2020.

[23] S. Hong, M. H. Lee, S. H. Kwon, and H. H. Chun, "A car test for the estimation of GPS/INS alignment errors," IEEE Transactions on Intelligent Transportation Systems, vol. 5, no. 3, pp. 208-218, 2004.

[24] W. Jiang, Y. Li, and C. Rizos, "On-the-fly Locata/inertial navigation system integration for precise maritime application," Measurement Science and Technology, vol. 24, no. 10, p. 105104, 2013.

[25] Y. Shen and G. Xu, "Simplified equivalent representation of GPS observation equations," GPS Solutions, vol. 12, pp. 99108, 2008.

[26] N. Carlson, "Federated filter for fault-tolerant integrated navigation systems," in Proceedings of the IEEE PLANS'88, Position Location and Navigation Symposium, Record, "Navigation into the 21st Century", pp. 110-119, Orlando, FL, USA, November 1988.

[27] N. Carlson, "Federated filter for computer-efficient, nearoptimal GPS integration," in Proceedings of the Position,
Location and Navigation Symposium-PLANS'96, pp. 306-314, Atlanta, GA, USA, April 1996.

[28] D.-H. Hwang, S. H. Oh, S. J. Lee, C. Park, and C. Rizos, "Design of a low-cost attitude determination GPS/INS integrated navigation system," GPS Solutions, vol. 9, no. 4, pp. 294-311, 2005.

[29] H. Lee, J. Lee, K. Yong, and G. Chan, "Modeling quaternion errors in SDINS: computer frame approach," IEEE Transactions on Aerospace and Electronic Systems, vol. 34, no. 1, pp. 289-300, 1998.

[30] Y. Tang, Y. Wu, M. Wu, W. Wu, X. Hu, and L. Shen, "INS/ GPS integration: global observability analysis," IEEE Transactions on Vehicular Technology, vol. 58, no. 3, pp. 1129-1142, 2009.

[31] Y. Xiang, Y. Gao, J. Shi, and C. Xu, "Carrier phase-based ionospheric observables using PPP models," Geodesy and Geodynamics, vol. 8, no. 1, pp. 17-23, 2017. 